\title{
大功率固态照明用荧光陶瓷研究进展
}

彭星淋 ${ }^{1,2}$, 李淑星 ${ }^{3}$, 刘泽华 ${ }^{4}$, 姚秀敏 ${ }^{1,2}$, 解荣军 ${ }^{3}$, 黄政仁 ${ }^{1,2,4}$, 刘学建 ${ }^{1,2}$

(1. 中国科学院 上海硅酸盐研究所, 上海 200050; 2. 中国科学院大学 材料科学与光电技术学院, 北京 100049 ;

3. 厦门大学 材料学院, 厦门 361005; 4. 中国科学院 宁波材料技术与工程研究所, 宁波 315201)

摘 要: 固态照明具有功率大、亮度高、体积小、节能环保等优点, 已成为 21 世纪最有前景的照明技术。作为固态 照明关键材料, 苂光材料的性能直接决定固态照明器件的显色指数、流明效率和可靠性等技术参数。相较于荧光单 晶、荧光玻璃、荧光薄膜及量子阱，荧光陶瓷因具有优异的热学和光学性质及微观结构易调控等特点，被认为是综 合性能最优的大功率固态照明用荧光材料。未来, 荧光陶瓷将在汽车大灯、户外照明、激光电视、激光影院等领域 得到更广泛的应用和发展, 具有广阔的市场前景。本文探讨了大功率固态照明用苂光陶瓷的设计原则, 重点介绍了 目前研究相对较多的氧化物苂光陶瓷(主要指钎铝石榴石结构)和氮(氧)化物苂光陶瓷的研究进展, 最后对大功率固 态照明用荧光陶瓷的未来发展方向进行了展望。

关 键 词: 固态照明; 苂光陶瓷; 钎铝石榴石; 氮(氧)化物; 综述

中图分类号: TQ174 文献标志码: A

\section{Phosphor Ceramics for High-power Solid-state Lighting}

\author{
PENG Xinglin $^{1,2}$, LI Shuxing ${ }^{3}$, LIU Zehua ${ }^{4}$, YAO Xiumin ${ }^{1,2}$, XIE Rongjun ${ }^{3}$, \\ HUANG Zhengren ${ }^{1,2,4}$, LIU Xuejian ${ }^{1,2}$
}

(1. Shanghai Institute of Ceramics, Chinese Academy of Sciences, Shanghai 200050, China; 2. College of Materials Science and Opto-Electronic Technology, University of Chinese Academy of Sciences, Beijing 100049, China; 3. College of Materials, Xiamen University, Xiamen 361005, China; 4. Ningbo Institute of Materials Technology and Engineering, Chinese Academy of Sciences, Ningbo 315201, China)

\begin{abstract}
Due to high power, high brightness, small size, energy saving, and environment friendliness, solid-state lighting has been becoming the most promising lighting technology in this century. As the key material of solid-state lighting, the luminescent properties of phosphors directly determine the crucial parameters such as the color rendering index, luminous efficacy and reliability of solid-state lighting devices. Compared with single crystals, phosphor glasses, phosphor films and quantum-well LEDs, phosphor ceramics have become the most excellent phosphor materials for high-power solid-state lighting due to its excellent thermal and optical properties and easy control of microstructure. In the future, phosphor ceramics are expected to be more widely used and developed in automotive headlights, outdoor lighting, laser TVs, laser cinema projectors, and other fields, and have a broad market prospect. In this review, design principles of high-power solid-state lighting phosphor ceramics are put forward firstly, and then research progress of oxide phosphor ceramics (mainly referring to $\mathrm{Y}_{3} \mathrm{Al}_{5} \mathrm{O}_{12}$ ) and
\end{abstract}

收稿日期：2020-11-12；收到修改稿日期：2020-12-24; 网络出版日期：2021-03-01

基金项目: 国家重点研发计划(2018YFB0406700) National Key R\&D Program of China (2018YFB0406700)

作者简介: 彭星淋(1995-), 男, 博士研究生. E-mail: pengxinglin@student.sic.ac.cn PENG Xinglin(1995-), male, PhD candidate. E-mail: pengxinglin@student.sic.ac.cn

通信作者: 刘学建, 研究员. E-mail: xjliu@mail.sic.ac.cn; 李淑星, 讲师. E-mail: lishuxing@xmu.edu.cn LIU Xuejian, professor. E-mail: xjliu@mail.sic.ac.cn; LI Shuxing, lecturer. E-mail: lishuxing@xmu.edu.cn 
nitrogen/oxynitride phosphor ceramics is reviewed mainly. Finally, the development of phosphor ceramics for high-power solid-state lighting is prospected.

Key words: solid-state lighting; phosphor ceramics; yttrium aluminum garnet; nitrogen/oxynitrides; review

固态照明是一种基于半导体芯片的全新照明技 术, 具有光电转换效率高、节能、环保、全固态、 使用寿命长等优点, 被认为是 21 世纪的新型绿色光 源 ${ }^{[1]}$ 。根据不同的激发芯片, 固态照明分为发光二极 管(LED)照明和激光(LD)照明。LED 相比于传统的 苂光灯和白炽灯, 具有节能环保、亮度高、色域广 及寿命长等优点, 已广泛应用于室内照明、显示屏 及交通信号灯等低功率照明和显示领域。但在高功 率密度下, LED 存在难以解决的“效率下降”难题 ${ }^{[2]}$ 。 与 LED 相比, LD 不仅效率更高、亮度更强、照射距 离更远，而且克服了 LED 固有的高功率下“效率下 降”问题, 在汽车大灯、户外照明、激光影院等领域 有着广阔的应用前景 ${ }^{[3]}$ 。

对于 LD 照明, 很难由一种装置同时发射红绿 蓝(RGB)三色激光, 通过 RGB 三色激光混合方式合 成白光存在一定的困难 ${ }^{[4]}$ 。借鉴 LED 芯片和苂光粉 一起封装实现白光的方法, 采用蓝光 LD 或近紫外 $\mathrm{LD}$ 光源搭配苂光粉来实现白色 $\mathrm{LD}$ 照明, 受到科研 人员的广泛关注。传统 LED 封装用硅胶, 在用于 LD 封装时常常产生“黄化”问题, 苂光粉热导率很 低 $\left(0.1 \sim 0.2 \mathrm{~W} \cdot \mathrm{m}^{-1} \cdot \mathrm{K}^{-1}\right)$, 很容易因温度升高而产生 热猝灭。因此, LD 照明通常采用不同于 LED 的远程 封装技术，优选具有高热导率的荧光材料，从而保 证 LD 器件优异的热稳定性。

LD 照明用荧光材料主要包括荧光陶瓷、荧光单 晶、荧光玻璃、荧光薄膜及量子阱等。其中, 荧光 单晶因制备困难、周期长、成本高及相对较低的光 提取效率等缺点, 应用受到限制; 苂光玻璃的热导 率较低(约 $1 \mathrm{~W} \cdot \mathrm{m}^{-1} \cdot \mathrm{K}^{-1}$ ), 在较低功率密度下易产生 发光饱和; 苂光薄膜存在致密度低及表面润湿不匹 配等不足; 量子阱则存在量子效率、光提取效率和 热导率偏低等问题 ${ }^{[3]}$ 。相比之下, 荧光陶瓷具有优异 的热学(热导率和热稳定性高)、光学(量子效率和光 提取效率高)性能及微观结构易调控等特点, 成为 综合性能最佳和最有应用潜力的大功率固态照明用 苂光材料 ${ }^{[3,5]}$ 。

目前，已有大功率固态照明用苂光材料及苂光 玻璃的综述报道, 尚缺乏荧光陶瓷研究进展的专题 综述。基于此，本文探讨了大功率固态照明用荧光 陶瓷的设计原则, 重点综述了研究相对较多的氧化 物荧光陶瓷(主要指钎铝石榴石结构)和氮(氧)化物
荧光陶瓷的近期研究进展, 最后展望了大功率固态 照明用苂光陶瓷的未来发展方向。

\section{1 大功率固态照明用荧光陶瓷的设计 原则}

大功率固态照明器件的应用环境非常苛刻, 对 荧光材料的光学、热学及机械性能都提出了更高的 要求。为满足大功率固态照明、特别是激光照明的 实际应用需求，亟需对苂光陶瓷进行剪裁设计和结 构优化，使其具有优异的发光饱和特性、较高的光 提取效率和一致性、较高的显色指数及良好的机械 性能 ${ }^{[3]}$ 。

\section{1 发光饱和特性}

随着激光功率密度的升高, 发光材料的光通量 出现先增加后保持不变甚至显著降低的现象称为发 光饱和, 是激光照明苂光材料的重要特性之一。发 光饱和分为热饱和与光饱和。其中, 光饱和的原因 有两种: 1)发光中心的数量有限, 随着激发光子数 的增加，没有多余的发光中心吸收更多的入射光子; 2)发光中心的衰减时间长, 间接导致发光中心的饱 和。例如相比于寿命较长的 $\mathrm{Eu}^{2+}, \mathrm{Ce}^{3+}$ 发光中心的 衰减时间较短 $(20 \sim 60 \mathrm{~ns}), \mathrm{Ce}^{3+}$ 通常在更高的功率 密度下才会发生发光饱和 ${ }^{[6]}$ 。而热饱和机理较为复 杂，一般可从产热、散热及耐热三方面解释，分别 阐述如下。

产热与吸收率、内量子效率及斯托克斯位移有 关，其定量关系如式(1)所示 ${ }^{[7]}$ :

$$
\mathrm{GH}=A \cdot P_{\mathrm{in}}\left(1-\frac{\mathrm{IQE} \cdot \lambda_{\mathrm{ex}}}{\lambda_{\mathrm{em}}}\right)
$$

其中, $\mathrm{GH}$ 为产热, $A$ 为吸收率, $P_{\mathrm{in}}$ 为入射激光功率, IQE 为内量子效率, $\lambda_{\mathrm{ex}}$ 表示激发波长, $\lambda_{\mathrm{em}}$ 为发射 波长。

从式(1)可知, 在入射激光功率保持不变的情况 下，吸收率越高，内量子效率越低，斯托克斯位移 (激发和发射波长的差值)越大，则产热越多，发光 饱和阈值越低。具体来说: 1)吸收率随发光中心浓度 增大而升高。以 $\mathrm{Y}_{3} \mathrm{Al}_{5} \mathrm{O}_{12}: \mathrm{Ce}^{3+}(\mathrm{YAG}: \mathrm{Ce})$ 荧光陶瓷为 例, 随着 $\mathrm{Ce}^{3+}$ 浓度增大, 吸收率升高, 产热增加, 导 致发光饱和阈值降低 ${ }^{[8]}$ 。2)提高内量子效率可减少 
无辐射跃迁过程的产热。需要指出的是, 量子效率 越低, 产热越高, 则材料温度越高, 温度升高又进 一步降低量子效率, 由此引发恶性循环。内量子效 率与荧光材料的晶体结构、发光中心浓度及种类等 本征特性有关。以 $\mathrm{Gd}_{3} \mathrm{Al}_{4} \mathrm{GaO}_{12}: \mathrm{Ce}(\mathrm{GAGG})$ 荧光陶 瓷为例, 随着 $\mathrm{Ga}^{3+}$ 浓度增大, 晶格占位发生改变, 内量子效率下降，产热增多 ${ }^{[8]}$ 。3)小斯托克斯位移可 以降低波长转换过程的产热。这意味着发射波长较 短的苂光陶瓷一般具有较少的产热和相对较高的发 光饱和阈值，例如绿色 $\mathrm{Lu}_{3} \mathrm{Al}_{5} \mathrm{O}_{12}: \mathrm{Ce}^{3+}$ (LuAG:Ce)、 黄色 YAG: Ce 和红色 $\mathrm{CaAlSiN}_{3}$ : $\mathrm{Eu}$ 荧光陶瓷的发射 波长分别约为 $520 、 540$ 和 $650 \mathrm{~nm}$, 对应的发光饱和 阈值分别为 $49 、 31.94$ 以及 $1.5 \mathrm{~W} \cdot \mathrm{mm}^{-2[6,8]}$ 。

散热与热导率、热管理及几何结构有关。具体 来说: 1)苂光材料热导率越高, 散热越好。YAG:Ce 的热导率约为 9 14 W $\cdot \mathrm{m}^{-1} \cdot \mathrm{K}^{-1}$, 通过引入较高热导 率的 $\mathrm{Al}_{2} \mathrm{O}_{3}\left(32 \sim 35 \mathrm{~W} \cdot \mathrm{m}^{-1} \cdot \mathrm{K}^{-1}\right)$ 制备出 $\mathrm{Al}_{2} \mathrm{O}_{3}-\mathrm{YAG}: \mathrm{Ce}$ 复相苂光陶瓷, 可以改善散热效果, 从而提高发光 饱和阈值 ${ }^{[9-12]}$ 。2)有效的热管理可以提高散热。例如: 将荧光陶瓷与高导热的 $\mathrm{Al}$ 基板组合利用热传导来散 热 ${ }^{[13-14]}$, 或采用旋转荧光色轮来促进散热 ${ }^{[14]}$ 。3) 几何 结构对散热的影响较复杂。一方面, 荧光材料的吸 收率随样品厚度的增加而增大, 产热随之增加; 另 一方面，激光光斑的温度分布曲线随厚度的增加而 展宽，有利于散热 ${ }^{[8]}$ 。

耐热与苂光材料的热猝灭直接相关, 而热猝灭 又受材料热激活能及掺杂元素的影响。热激活能 $\Delta E$ 是指激发态和基态位形曲线的交叉点与激发态最低 能级之间的能量差, 热激活能越高, 苂光材料的热 猝灭越小, 耐热性越佳。Xu 等 ${ }^{[8]}$ 的研究表明, 采用 $\mathrm{Lu}$ 取代 $\mathrm{Y}$ 可以降低 YAG 的热猝灭, 提高其耐热性, 进而提高材料的发光饱和阈值, 而采用 $\mathrm{Gd}$ 取代 $Y$ 则产生相反的结果。

\section{2 光提取效率和一致性}

激光是一种具有比 LED 功率密度更高的点光 源, 因此 LD 照明需要更有效的散射中心来增加光 的吸收, 使蓝色激光束均匀化 ${ }^{[14]}$ 。散射中心对提高 光的吸收和提取效率、均匀混合泵入光和转换光以 及限制光斑直径实现高亮度照明具有重要作用 ${ }^{[3]}$ 。 未经散射和转化的入射蓝光会直接穿过苂光材料 造成“黄环效应”(Yellow Ring Effect), 影响出光一 致性 ${ }^{[15]}$ 。提高光提取效率和一致性的关键在于提高 苂光材料对光的散射，常见方法有引入气孔 ${ }^{[14,16]}$ 、 引入不发光的第二相 ${ }^{[17-18]}$ 、表面粗糙化 ${ }^{[19-20]}$ 及表面 修饰 ${ }^{[21-22]}$ 等。

Zhang 等 ${ }^{[16]}$ 采用不同烧结温度制备了不同气孔
率的 LuAG:Ce 荧光陶瓷，并研究了气孔率对荧光陶 瓷发光性能的影响。研究结果表明, 经 $1650{ }^{\circ} \mathrm{C}$ 烧结 后含 $2.88 \%$ 气孔率的苂光陶瓷, 发光强度最高, 在 $450 \mathrm{~nm}$ 蓝光照射下的光通量随入射光功率密度增 大而线性增大, 流明效率达 $200 \mathrm{~lm} / \mathrm{W}$, 在动态和静 态模式下的转换效率分别高达 $50.2 \%$ 和 $44.4 \%$, 这 表明引入适量气孔有利于提升发光性能。Zheng 等 ${ }^{[14]}$ 采用造孔剂方法制备了气孔尺寸约 $2 \mu \mathrm{m}$ 、体积分数 8.0\% 24.6\%的 YAG:Ce 荧光陶瓷。研究发现通过气 孔可调控荧光陶瓷的散射性能, 有助于限制光斑直 径、提高亮度、降低光束发散度, 最终获得了超高 亮度和一致性的白光, 提高了光提取效率。这似乎 颠覆了人们对荧光陶瓷的传统认知, 即荧光陶瓷应 是透明或半透明的。Zheng 等制备的含有体积分数 15\%气孔的 YAG:Ce 苂光陶瓷是不透明的, 但却获 得了 $7199 \mathrm{~lm}$ 的超高光通量, 高于目前已报道的所 有 YAG:Ce 苂光陶瓷。由此可见，在激光照明用苂 光陶瓷中引入合适的气孔来提高光散射, 进而提高 其发光性能的效果十分显著。

与引入气孔类似, 通过引入不发光的第二相也 可以提高荧光陶瓷的散射, 这是由于引入第二相作 为散射中心改变了激发光在陶瓷中的传播路径, 从 而提高了光提取效率 ${ }^{[23-24]}$ 。例如, Liu 等 ${ }^{[18]}$ 制备了性 能优异的 $\mathrm{MgAl}_{2} \mathrm{O}_{4}-\mathrm{GdYAG}: \mathrm{Ce}$ 复相苂光陶瓷, 得益 于 $\mathrm{MgAl}_{2} \mathrm{O}_{4}$ 第二相粒子对光的散射, 改变了光线传 播路径，增加了荧光陶瓷对蓝光的吸收和对黄光的 提取, 其示意图如图 1 所示。除此之外, 更多的研究 则是添加 $\mathrm{Al}_{2} \mathrm{O}_{3}$ 作为基质来提高荧光陶瓷的光提取 效率 ${ }^{[10-12]}$ 。

Wagner 和 Sun 等 ${ }^{[19-20]}$ 研究了荧光陶瓷表面粗粘 度对发光性能的影响，发现粗糙表面有利于减弱 “全内反射效应”，从而提高其光提取效率。Sun 等 ${ }^{[19]}$ 通过机械研磨处理制备了具有不同表面粗粘度的 YAG:Ce 荧光陶瓷，由于全反射效应在粗䊁表面作

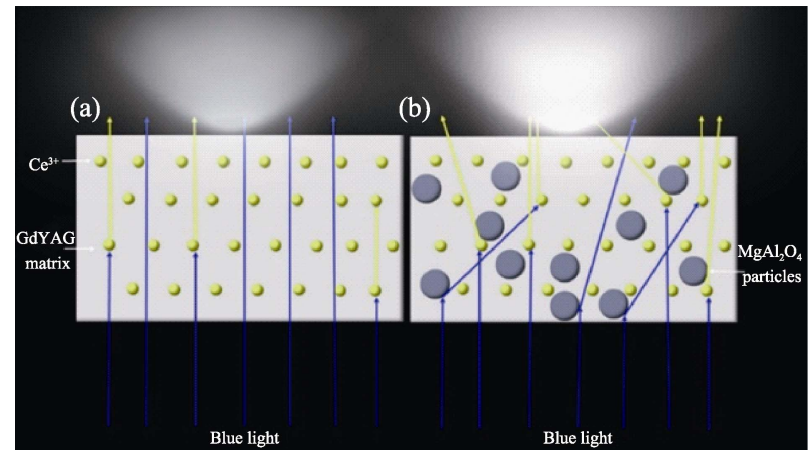

图 1 单相陶瓷(a)和复相陶瓷(b)的光传播和转化示意图 ${ }^{[18]}$ Fig. 1 Schematic of light conversion and propagation in monolithic (a) and composite (b) ceramics $^{[18]}$ 
用下减弱, 这使得更多的转换黄光可以透过陶瓷表面, 通过控制陶瓷的厚度和表面粗糙度, 显著提高了光提 取效率和转换效率, 最终获得流明效率为 $85.57 \mathrm{~lm} / \mathrm{W}$, 色温为 $4358 \mathrm{~K}$ 的苂光陶瓷。Wagner 等 ${ }^{[20]}$ 通过砂纸 打磨处理制备出表面粗粘度 $R_{\mathrm{a}}$ 在 30 1570 nm 范围 变化的 YAG:Ce 苂光陶瓷, 发光强度和外量子效率 随 $R_{\mathrm{a}}$ 的增大分别增大了 $60 \%$ 和 $100 \%$ 。

通过在苂光陶瓷表面制备二维光子晶体等表面 修饰技术也可以提高苂光陶瓷的光提取效率和光一 致性。例如, Park 等 ${ }^{[21]}$ 通过在 YAG:Ce 苂光陶瓷表 面制备纳米孔状结构的 $\mathrm{SiN}_{x}$ 二维光子晶体涂层, 将 蓝光吸收率和黄光提取效率分别提高了 $34 \%$ 和 $29 \%$, 使苂光陶瓷发射强度提高了 $72 \%$ ，流明效率提高了 $55 \%(44.2 \rightarrow 68.8 \mathrm{~lm} / \mathrm{W})$ 。随后, 该研究团队 ${ }^{[22]}$ 通过 在 YAG:Ce 苂光陶瓷表面制备纳米碗状结构的 $\mathrm{TiO}_{2}$ 二维光子晶体涂层, 进一步将苂光陶瓷的流明效率 提高了 $54 \%$, 达到 $119 \mathrm{~lm} / \mathrm{W}$ 。然而, 苂光陶瓷表面 修饰的工艺非常复杂, 包括薄膜沉积、光刻掩模、 自组装、硬掩模沉积和干蚀刻或湿蚀刻等过程, 这 极大制约了该方法的实际应用 ${ }^{[19]}$ 。

\section{3 显色指数}

目前大功率固态照明用苂光陶瓷普遍存在的难 题之一是显色指数偏低, 这主要是因为苂光陶瓷往 往缺少长波段的红色成分。LD 比 LED 的发射峰更 加尖锐, 半峰全宽更窄 $(L D \approx 1 \mathrm{~nm}, \mathrm{LED} \approx 20 \mathrm{~nm})$, 因此激光照明的显色指数往往更低(约 60$)^{[3]}$ 。通过 调控基质化学成分 ${ }^{[25-27]}$ 、改变发光中心种类 ${ }^{[28-31]}$ 、 复合红色荧光材料 ${ }^{[32-35]}$ 等方法来优化苂光陶瓷的发 射峰位和半峰全宽、进而提高显色指数, 成为近年 来苂光陶瓷材料研究的重点方向之一。具体研究进 展将在第 2 部分详细阐述。

\section{4 机械性能}

对于大功率固态照明尤其是激光照明用苂光陶 瓷而言, 在使用过程中长时间经受高能光束的照射, 产生巨大的热应力, 致使苂光材料容易开裂或损坏, 因此良好的机械性能对于提高照明器件的稳定性和 使用寿命至关重要。目前有关苂光陶瓷机械性能研 究的报道并不多 ${ }^{[12,36-37]}$ 。为提高照明器件在实际使 用过程中的稳定性和寿命, 荧光陶瓷应具有高强 度、高断裂㓞性及良好的抗热震性能。

\section{2 大功率固态照明用荧光陶瓷的材料 体系}

近年来, 研究人员在大功率固态照明用苂光陶
瓷领域开展了卓有成效的研究, 涉及的材料体系主 要包括氧化物荧光陶瓷(钎铝石榴石体系)和氮(氧) 化物荧光陶瓷两大类, 具体研究进展综述如下。

\section{1 氧化物荧光陶瓷}

YAG:Ce 是目前技术最成熟、应用最广泛、价 格最低廉的荧光粉材料体系, YAG:Ce 苂光粉和蓝光 LED 芯片的组合也是目前最普遍采用的白光实现 方式。YAG 钎铝石榴石结构属于典型的立方晶系, 没有双折射效应, 所以, 理论上可以制成高透过率 的透明陶瓷。科研人员以 YAG:Ce 苂光粉为原料来 烧结制备 YAG:Ce 苂光陶瓷 ${ }^{[38-39]}$, 得到的材料往往 存在光提取效率、热导率和显色指数较低以及色温 偏高等缺点 ${ }^{[26,40]}$ 。目前, 多采用以下四种方法来解 决上述问题: 通过引入不发光第二相来解决光提取 效率和热导率偏低问题, 通过调整基质化学组成、 发光中心种类或复合红色荧光材料等方式来解决显 色指数和色温方面的问题。具体研究进展分述如下。

\subsection{1 引入不发光第二相}

引入不发光第二相的目的一般是为了提高光提 取效率和(或)热导率, 其中引入不发光第二相提高 光提取效率的机理上文已讨论(1.2 节，图 1)。而通 过引入高热导基质来提高热导率的主要理论基础是 双组分麦克斯韦 - 加内特模型 (Two-component Maxwell Garnett Model), 如式(2)所示:

$$
K_{\text {eff }}=K_{1} \frac{K_{2}(1+2 V)-K_{1}(2 V-2)}{K_{1}(2+V)+K_{2}(1-V)}
$$

其中 $K_{\mathrm{eff}} 、 K_{1}$ 和 $K_{2}$ 分别指复相陶瓷、连续相和分散 相的热导率, $V$ 指分散相的体积分数 ${ }^{[41]}$ 。

对于钎铝石榴石体系荧光陶瓷来说, 最常见的 基质材料为 $\mathrm{Al}_{2} \mathrm{O}_{3}$, 这是由于: 1 ) $\mathrm{Al}_{2} \mathrm{O}_{3}$ 热导率(32 $\left.35 \mathrm{~W} \cdot \mathrm{m}^{-1} \cdot \mathrm{K}^{-1}\right)$ 高于 $\left.\mathrm{YAG}\left(9 \sim 14 \mathrm{~W} \cdot \mathrm{m}^{-1} \cdot \mathrm{K}^{-1}\right) ; 2\right) \mathrm{Al}_{2} \mathrm{O}_{3}$ 热膨胀系数 $\left(8.4 \times 10^{-6} \mathrm{~K}^{-1}\right)$ 与 $\mathrm{YAG}\left(8.0 \times 10^{-6} \mathrm{~K}^{-1}\right)$ 相近; 3) $\mathrm{Al}_{2} \mathrm{O}_{3}$ 和 $\mathrm{YAG}$ 之间不发生化学反应, 化学相容性 好; 4) $\mathrm{Al}_{2} \mathrm{O}_{3}$ 禁带宽度为 7 8 eV, 本征吸收位于远紫 外区域, 不会对 YAG:Ce ${ }^{3+}$ 苂光材料在可见光区的吸 收产生影响; 5)尽管 $\mathrm{Al}_{2} \mathrm{O}_{3}$ 属于六方晶系, 存在双 折射效应, 但仍可制备成半透明陶瓷, 且烧结温度 较低; 6) $\mathrm{Al}_{2} \mathrm{O}_{3}$ 成本较低 ${ }^{[10]}$ 。因此, $\mathrm{Al}_{2} \mathrm{O}_{3}-\mathrm{YAG}: \mathrm{Ce}$ 复 相苂光陶瓷成为目前研究最广泛和最深入的大功率 固态照明用苂光陶瓷材料 ${ }^{[9-12]}$ 。例如, Wang 等 ${ }^{[11]}$ 制 备了可用于高亮度激光照明透射模式的 “热自管 理” $\mathrm{Al}_{2} \mathrm{O}_{3}-\mathrm{YAG}: \mathrm{Ce}$ 复相苂光陶瓷, 即通过引入 $\mathrm{Al}_{2} \mathrm{O}_{3}$ 提高热导率从而避免安装散热片。如图 2 所示, 复 相苂光陶瓷具有致密的显微结构, 热导率最高达 $32.5 \mathrm{~W} \cdot \mathrm{m}^{-1} \cdot \mathrm{K}^{-1}($ 图 $2(\mathrm{~d}))$ 。热导率虽然随温度升高逐 

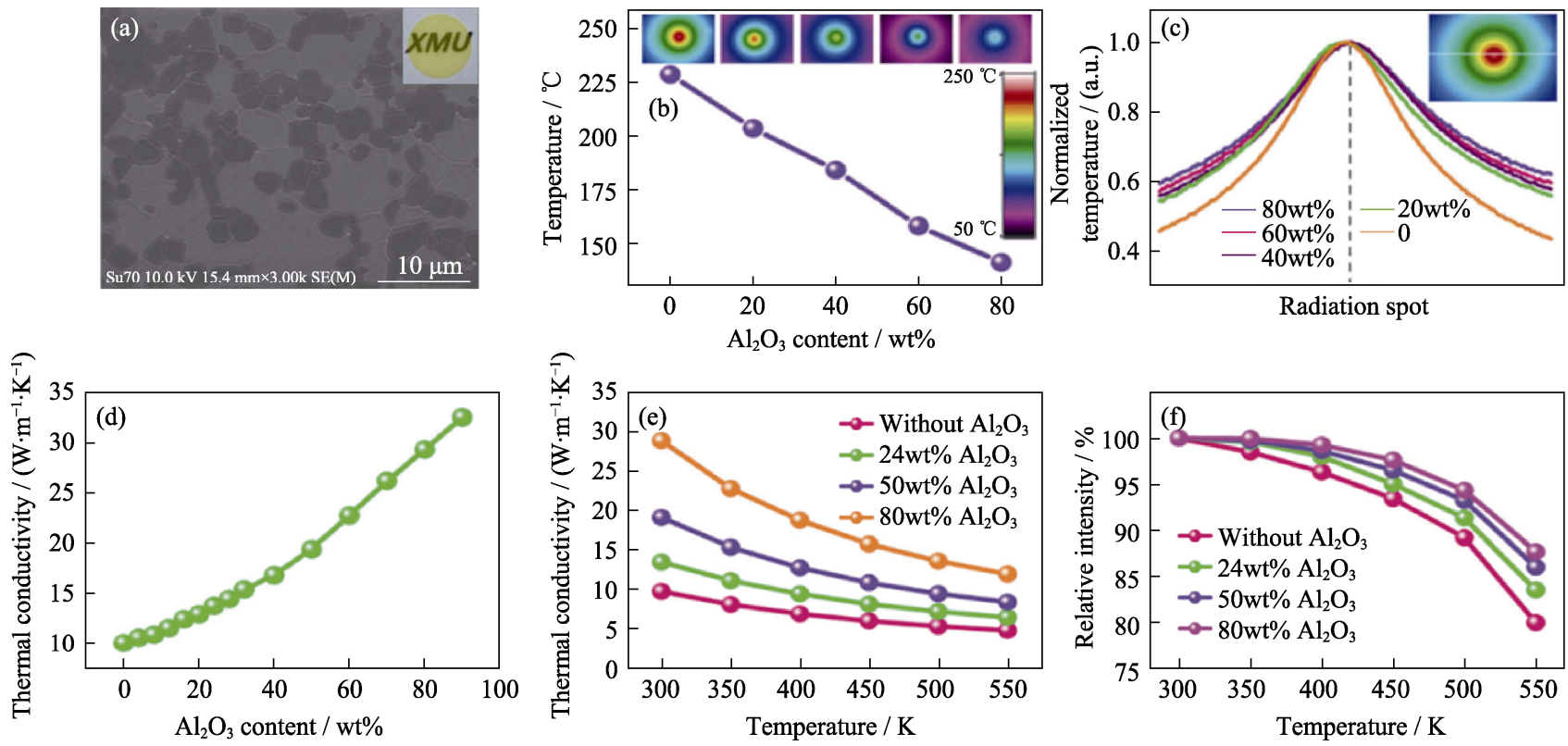

图 $2 \mathrm{Al}_{2} \mathrm{O}_{3}-\mathrm{YAG}: \mathrm{Ce}$ 复相苂光陶瓷 ${ }^{[11]}$

Fig. $2 \quad \mathrm{Al}_{2} \mathrm{O}_{3}$-YAG:Ce composite phosphor ceramics ${ }^{[11]}$

(a) SEM image of the $\mathrm{Al}_{2} \mathrm{O}_{3}$-YAG:Ce composite ceramics; (b) Laser irradiation spot temperature of the ceramics varies with different $\mathrm{Al}_{2} \mathrm{O}_{3}$ contents; (c) Temperature distribution curves; (d) Thermal conductivity as a function of $\mathrm{Al}_{2} \mathrm{O}_{3}$ content; (e) Thermal conductivity as a function of the temperature; (f) Temperature-dependent integrated emission intensity of the composite ceramics Colorful figures are available on website

渐下降(图 2(e)), 但在 $500 \mathrm{~K}$ 的发光强度仍能保持室 温的 $90 \%$ 以上(图 2(f)); 随 $\mathrm{Al}_{2} \mathrm{O}_{3}$ 含量增加, 激光辐 照光斑的温度逐渐降低(图 2(b)), 且温度梯度逐渐 减小(图 2(c)), 非常利于散热; 通过调控 $\mathrm{Al}_{2} \mathrm{O}_{3}$ 含量 及样品厚度, 在 $20.1 \mathrm{~W} \cdot \mathrm{mm}^{-2}$ 的功率密度下获得了 光通量为 $1367 \mathrm{~lm}$ 、流明效率为 $135.32 \mathrm{~lm} / \mathrm{W}$ 、亮度 为 $982 \mathrm{Mcd} \cdot \mathrm{m}^{-2}$ 的 $\mathrm{Al}_{2} \mathrm{O}_{3}-\mathrm{YAG}: \mathrm{Ce}$ 复相苂光陶瓷。

除 $\mathrm{Al}_{2} \mathrm{O}_{3}$ 外, 部分研究还引入了 $\mathrm{CaF}_{2}$ 、 $\mathrm{MgAl}_{2} \mathrm{O}_{4}$ 、羟基磷灰石(Hydroxyapatite, $\mathrm{HA}$ )等作为 基质材料 ${ }^{[17-18,42]}$ 。例如, Huang 等 ${ }^{[17]}$ 以水热合成的 $\mathrm{HA}$ 介孔纳米棒和 YAG:Ce 苂光粉为原料, 采用 SPS 烧结工艺在较低温度下 $\left(850{ }^{\circ} \mathrm{C}\right)$ 制备了外量子效率 为原始苂光粉 $90 \%$ 以上的 HA-YAG:Ce 复相苂光陶 瓷; 在 SPS 高压下, HA 产生明显的取向生长, 形成 纳米波片机制，消除了双折射的影响; 通过晶粒尺 寸调控和纳米波片结构设计, 巧妙利用瑞利散射进 一步提高了蓝光转换效率, 从而获得了发光效率达 $170 \mathrm{~lm} / \mathrm{W}$ 的透射式 WLED 器件。 Gu 等 ${ }^{[42]}$ 采用热压烧 结工艺在较低温度下 $\left(700{ }^{\circ} \mathrm{C}\right)$ 制备出 $\mathrm{CaF}_{2}-\mathrm{YAG}: \mathrm{Ce}$ 复 相荧光陶瓷; 引入 $\mathrm{CaF}_{2}$ 提高了光提取效率, 内量子 效率达 $83.2 \%$, 具有较低的热猝灭特性 $\left(150{ }^{\circ} \mathrm{C}\right.$ 下发 光强度仅损失 $9 \%$ ); 相应 WLEDs 器件的光通量达 到 $359.71 \mathrm{~m}$ 。

\subsection{2 调整基质化学组成}

$\mathrm{Y}_{3} \mathrm{Al}_{5} \mathrm{O}_{12}(\mathrm{YAG})$ 属于立方晶系, 空间群为 $\mathrm{Ia} \overline{3} \mathrm{~d}$, 分子式可以写为 $A_{3} B_{2} C_{3} O_{12}$, 其中, $A 、 B 、 C$ 代表
三个不同的格位。 $\mathrm{Y}^{3+}$ 离子处于 $\mathrm{A}$ 格位，与周围的八 个 $\mathrm{O}^{2-}$ 离子组成十二面体; $\mathrm{B}$ 和 $\mathrm{C}$ 的位置被 $\mathrm{Al}^{3+}$ 离子 所占据, 分别与周围的六个和四个 $\mathrm{O}^{2-}$ 离子构成八 面体和四面体 ${ }^{[43-44]}$ 。对于大部分石榴石体系苂光陶 瓷来说, 发光中心一般选择 $\mathrm{Ce}^{3+}$, 当 $\mathrm{Ce}^{3+}$ 的晶体场 受到晶体结构变化的影响时, 其发光性能也会由 于晶体场䢃裂效应(Crystal-field Splitting)而发生变 化 ${ }^{[25-26]}$ 。一般情况下, 离子在十二面体位置上(A 格 位)的直径增大或八面体位置上(B 格位)的直径减小 都会导致晶体场强增大，发射光谱红移，从而提高 显色指数和降低相关色温 ${ }^{[25,44]}$ 。如表 1 所示, 目前 已报道的石榴石体系荧光陶瓷中, 取代 YAG:Ce 中 十二面体位置 $(\mathrm{A}$ 格位 $) \mathrm{Y}^{3+}$ 的离子种类有 $\mathrm{Tb}^{3+} 、 \mathrm{Gd}^{3+}$ 、 $\mathrm{Lu}^{3+} 、 \mathrm{Mg}^{2+} 、 \mathrm{Sc}^{3+}$ 等, 取代八面体位置 $(\mathrm{B}$ 格位 $) \mathrm{Al}^{3+}$ 的离子种类有 $\mathrm{Ga}^{3+} 、 \mathrm{Mg}^{2+} 、 \mathrm{Sc}^{3+}$ 等, 取代四面体位 置 $(\mathrm{C}$ 格位 $) \mathrm{Al}^{3+}$ 的离子种类有 $\mathrm{Si}^{4+}$ 等 ${ }^{[25-27]}$ 。占据十二 面体位置的 $\mathrm{Gd}^{3+}$ 和 $\mathrm{Tb}^{3+}$ 的离子半径大于 $\mathrm{Y}^{3+}$, 因此 在 $\mathrm{YAG}$ 中掺杂 $\mathrm{Gd}^{3+}$ 或 $\mathrm{Tb}^{3+}$ 会引起晶体场强增大、 光谱红移, 且 $\mathrm{Gd}^{3+}$ 半径大于 $\mathrm{Tb}^{3+}$, 掺杂 $\mathrm{Gd}^{3+}$ 引起的 光谱红移更显著。有趣的是, 八面体位置上的 $\mathrm{Mg}^{2+}$ 半径大于 $\mathrm{Al}^{3+}$, 采用 $\mathrm{Mg}^{2+}$ 取代 $\mathrm{Al}^{3+}$ 理应引起光谱蓝 移, 但 $\mathrm{Mg}-\mathrm{Si}$ 共掺却引起光谱红移。Tian 等 ${ }^{[27]}$ 采用 位形坐标曲线分析了 $\mathrm{Y}_{3} \mathrm{Mg}_{x} \mathrm{Al}_{5-2 x} \mathrm{Si}_{x} \mathrm{O}_{12}$ : $\mathrm{Ce}$ (YMASG: $\mathrm{Ce})$ 荧光陶瓷红移的主要原因是斯托克斯位移增大。 可以认为, 石榴石体系苂光陶瓷中的 $\mathrm{Mg}-\mathrm{Si}$ 共掺杂会 同时影响晶体场䢃裂和斯托克斯位移, 且两 
表 1 石榴石体系荧光陶瓷不同格位掺杂离子及离子半径汇总表

Table 1 Doping ions and ionic radii of garnet phosphor ceramics at different lattice positions

\begin{tabular}{cccc}
\hline Doped ions & Ion radius/nm & Occupied lattice & Ref. \\
\hline $\mathrm{Y}^{3+}$ & 0.1019 & $\mathrm{~A}$ & {$[38]$} \\
$\mathrm{Gd}^{3+}$ & 0.1053 & $\mathrm{~A}$ & {$[26]$} \\
$\mathrm{Tb}^{3+}$ & 0.104 & $\mathrm{~A}$ & {$[45]$} \\
$\mathrm{Lu}^{3+}$ & 0.0977 & $\mathrm{~A}$ & {$[46]$} \\
$\mathrm{Mg}^{2+}$ & 0.089 & $\mathrm{~A}$ & {$[27]$} \\
$\mathrm{Sc}^{3+}$ & 0.087 & $\mathrm{~A}$ & {$[43]$} \\
$\mathrm{Al}^{3+}$ & 0.0535 & $\mathrm{~B}$ & {$[38]$} \\
$\mathrm{Sc}^{3+}$ & 0.0745 & $\mathrm{~B}$ & {$[43]$} \\
$\mathrm{Mg}^{2+}$ & 0.072 & $\mathrm{~B}$ & {$[44]$} \\
$\mathrm{Ga}^{3+}$ & 0.062 & $\mathrm{~B}$ & {$[26]$} \\
$\mathrm{Al}^{3+}$ & 0.039 & $\mathrm{C}$ & {$[38]$} \\
$\mathrm{Si}^{4+}$ & 0.026 & $\mathrm{C}$ & {$[44]$} \\
\hline
\end{tabular}

Note: A stands for dodecahedron, B for octahedron and C for tetrahedron

者的影响趋势恰好相反, 斯托克斯位移增大导致的 光谱红移占主导作用, 因此晶体场䢃裂减小导致的 光谱蓝移现象被掩盖。

调整石榴石体系苂光陶瓷的化学组成, 主要是 为了通过增加晶体场䢃裂效应或者增大斯托克斯位 移来使光谱红移, 从而提高荧光陶瓷的显色指数, 降低相关色温。例如, Liu 等 ${ }^{[47]}$ 制备了 GAGG: Ce 苂
光陶瓷，引入 $\mathrm{Gd}^{3+}$ 使发射光谱红移，因此采用该苂 光陶瓷封装的 $\operatorname{COB}$ (Chips on Board)器件发出了相 关色温低于 $2800 \mathrm{~K}$ 的暖白光, 具有高达 $2100 \mathrm{~lm}$ 的 光通量, $388 \mathrm{~lm} / \mathrm{W}$ 的流明效率为目前已报道的大功 率 LED 照明器件的最高值。Chen 等 ${ }^{[25]}$ 制备了性能 优异的 $(\mathrm{Tb}, \mathrm{Gd})_{3} \mathrm{Al}_{5} \mathrm{O}_{12}: \mathrm{Ce}^{3+}$ (TGAG: $\mathrm{Ce}$ )苂光陶瓷, 通过引入 $\mathrm{Gd}^{3+}$ 取代十二面体位置的 $\mathrm{Tb}^{3+}$, 使晶体场 强度进一步增大, 光谱进一步红移, 进而提高显色 指数, 降低相关色温; 随着 $\mathrm{Gd}^{3+}$ 掺杂量的增加, 发 射峰位从 $550 \mathrm{~nm}$ 红移至 $570 \mathrm{~nm}$, 发光强度也大大 增加, 最终制备出相关色温 $3681 \mathrm{~K}$ 、显色指数 74.7 的暖白光固态照明器件。 $\mathrm{Du}$ 等 ${ }^{[44]}$ 通过在 $\mathrm{YAG}: \mathrm{Ce}$ 荧光陶瓷中共掺杂 $\mathrm{Mg}^{2+}$ 和 $\mathrm{Si}^{4+}$, 增大了 $\mathrm{Ce}^{3+}$ 的 $5 \mathrm{~d}$ 态能级晶体场䢃裂程度, 使发射光谱发生较大红移, 制备出性能优异的 YMASG: Ce 苂光陶瓷; 如图 3 所示, 随着 $\mathrm{Mg}-\mathrm{Si}$ 共掺杂含量提高 $(x=0 \rightarrow 2)$, YMASG: Ce 苂光陶瓷的发射峰位从 $533 \mathrm{~nm}$ 红移至 $598 \mathrm{~nm}$ (图 3(b)), 半峰全宽从 $115 \mathrm{~nm}$ 扩大到 $143 \mathrm{~nm}$ (图 3(c)); 发射峰位的红移和半峰全宽的增大都有 利于提高固态照明器件的显色指数, 色坐标向红色 区域位移也证实了 $\mathrm{Mg}-\mathrm{Si}$ 共掺杂可以有效降低色温 (图 3(d)); 和商用蓝色 LED 芯片封装后, 最终制备出 相关色温 $3762 \mathrm{~K}$ 的暖白光 LED 器件。最近, Yao 等 ${ }^{[5]}$ 利用真空烧结制备了 YAG:Ce 苂光陶瓷, 通过引入
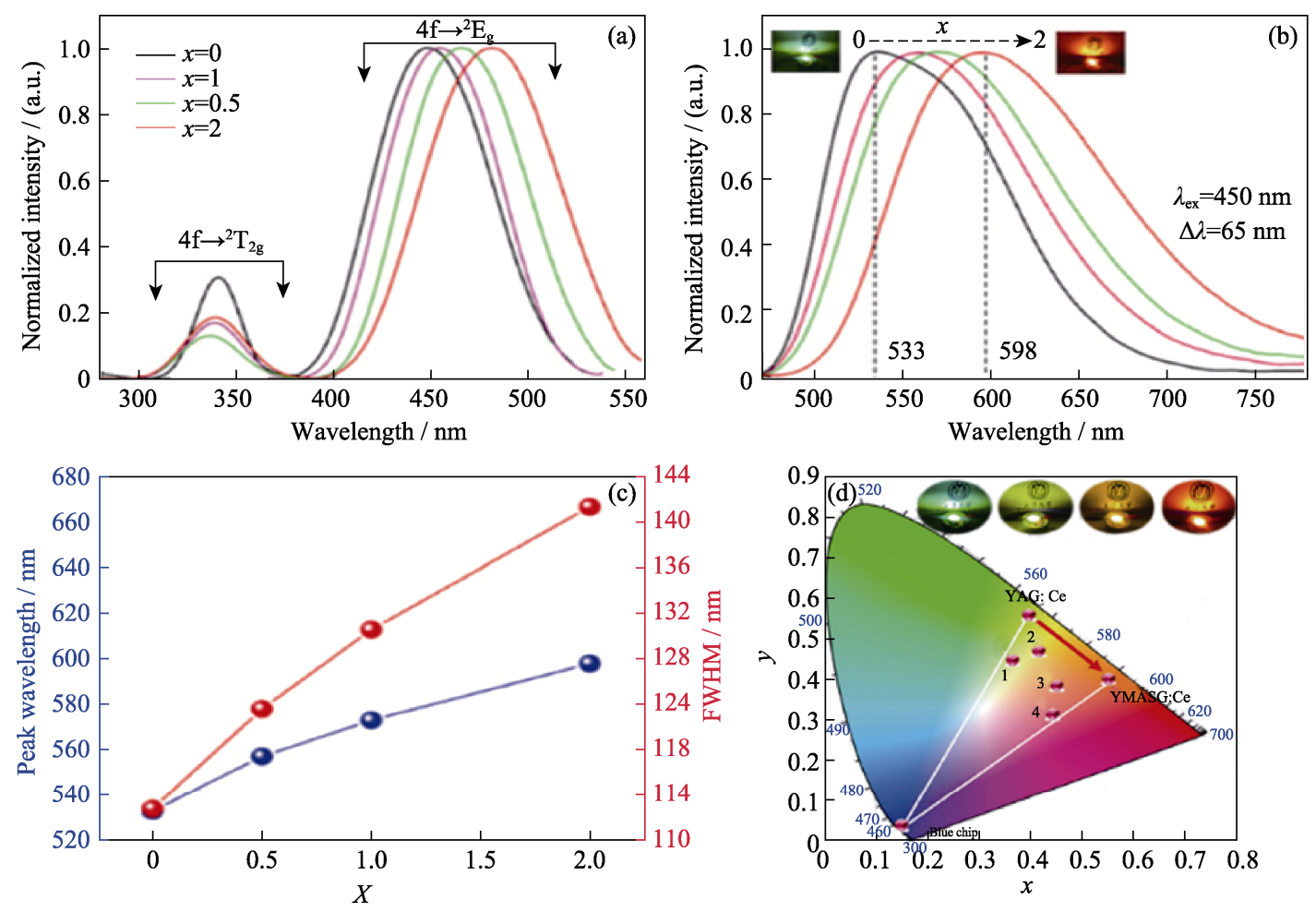

图 3 YMASG:Ce 荧光陶瓷 ${ }^{[44]}$

Fig. 3 YMASG:Ce phosphor ceramics ${ }^{[44]}$

(a) PLE spectra; (b) PL spectra; (c) Peak wavelength and FWHM; (d) Chromaticity color coordinates

Colorful figures are available on website 
纳米 $\mathrm{MgO}$ 和 $\mathrm{SiO}_{2}$ 并结合氧化处理消除了氧空位, 使流明效率从 $106 \mathrm{~lm} / \mathrm{W}$ 提高到 $223 \mathrm{~lm} / \mathrm{W}$, 达到了 目前已报道的 LD 照明器件的最高值, 优化后的荧 光陶瓷的显色指数和相关色温分别为 73 和 $7293 \mathrm{~K}$ 。

\subsection{3 调整发光中心种类}

石榴石体系苂光陶瓷最常见的发光中心是 $\mathrm{Ce}^{3+}$, 主要基于以下考虑: 1)按照选择定则, $4 \mathrm{f}^{\mathrm{n}} \rightarrow 4 \mathrm{f}^{\mathrm{n}-1} 5 \mathrm{~d}^{1}$ 跃迁是允许的, 这决定其具有高振子强度的特性 (强的吸收和发射)；2) $\mathrm{Ce}^{3+}$ 离子的 $5 \mathrm{~d}$ 能级暴露在晶 体场中，极易受局域配位环境的影响，通过改变基 质或者晶格结构可对其吸收和发射光谱产生较大的 影响, 具有可设计与调控的特点; 3)20 60 ns 的衰减 时间大大减弱了光饱和现象。YAG:Ce 是典型的黄 色荧光陶瓷，除了上文述及的通过调整基质化学成 分可以使发射光谱红移外, 还可以通过掺杂或者共 掺杂其他发光中心来引入红光成分, 从而提高显色 指数和降低相关色温。目前, 已报道的石榴石体系 苂光陶瓷的发光中心主要包括: $\mathrm{Ce}^{3+} 、 \mathrm{Dy}^{3+} 、 \mathrm{Mn}^{4+}$ 、 $\mathrm{Eu}^{3+} 、 \mathrm{Ce}^{3+} / \mathrm{Dy}^{3+}, \mathrm{Ce}^{3+} / \mathrm{Mn}^{2+}, \mathrm{Ce}^{3+} / \mathrm{Eu}^{3+}, \mathrm{Ce}^{3+} / \mathrm{Cr}^{3+} 、$ $\mathrm{Ce}^{3+} / \mathrm{Pr}^{3+} 、 \mathrm{Ce}^{3+} / \mathrm{Pr}^{3+} / \mathrm{Cr}^{3+}$ 等 ${ }^{[28-31]}$ 。例如, Ao 等 ${ }^{[28]}$ 制备 了 $\mathrm{YAG}: \mathrm{Ce}^{3+} / \mathrm{Mn}^{2+} / \mathrm{Si}^{4+}$ 荧光陶瓷，随 $\mathrm{Mn}^{2+}-\mathrm{Si}^{4+}$ 掺杂 量的增加, 显色指数逐渐提高 $(61.8 \rightarrow 82.5)$, 相关色 温也随之降低 $(5196 \mathrm{~K} \rightarrow 3870 \mathrm{~K})$, 但是流明效率却 大幅降低 $(115.08 \mathrm{~lm} / \mathrm{W} \rightarrow 14.00 \mathrm{~lm} / \mathrm{W})$ 。Feng 等 ${ }^{[29]}$ 制 备了 YAG: $\mathrm{Ce}^{3+} / \mathrm{Pr}^{3+} / \mathrm{Cr}^{3+}$ 苂光陶瓷, 如图 4 所示, 当 $\mathrm{Ce}^{3+}$ 和 $\mathrm{Pr}^{3+}$ 共掺杂时, 可以增加橘红色波段光的发 射; 当 $\mathrm{Ce}^{3+}$ 和 $\mathrm{Cr}^{3+}$ 共掺杂时, 可以增加深红色波段
光的发射; 当 $\mathrm{Ce}^{3+} 、 \mathrm{Pr}^{3+}$ 和 $\mathrm{Cr}^{3+}$ 共掺杂时, 荧光陶瓷 同时包含 530、609 和 $689 \mathrm{~nm}$ 等发射峰, 获得了 $500 \sim 750 \mathrm{~nm}$ 的超宽发射光谱范围, 这得益于 $\mathrm{Ce}^{3+} \rightarrow$ $\mathrm{Pr}^{3+}$ 以及 $\mathrm{Ce}^{3+} \rightarrow \mathrm{Cr}^{3+}$ 之间能量跃迁过程。有效补充的 红光成分, 使最终发光器件的显色指数高达 78 。

\subsection{4 复合红色荧光材料}

对于黄色/绿色石榴石体系荧光陶瓷而言，提高 显色指数和降低相关色温的更为直接的方法是复合 红色苂光材料。根据不同的复合方式，可分为均匀 复合、层状复合和薄膜复合三种类型 $[32-35,48-50]$ 。

均匀复合型苂光陶瓷是指把黄色荧光粉 (如

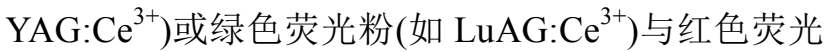
粉(如 $\mathrm{CaAlSiN}_{3}: \mathrm{Eu}^{2+} 、 \mathrm{~K}_{2} \mathrm{TiF}_{6}: \mathrm{Mn}^{4+}$ 等)均匀混合后制 备出的复相苂光陶瓷。例如, Krasnoshchoka 等 ${ }^{[48]}$ 将 $\mathrm{LuAG}: \mathrm{Ce}^{3+}$ 和 $\mathrm{Eu}^{2+}$ 掺杂的氮化物红粉均匀混合制备 出激光照明用复相苂光陶瓷, 其在高输入功率下, 色坐标发生了显著变化, 且发射光谱红移, 最终获 得了转换效率 $140.8 \mathrm{~lm} / \mathrm{W}$ 、显色指数 89.4 的复相荧 光陶瓷。该方法的局限性在于不同种类的荧光粉在 高温下容易发生界面反应乃至化学反应，使荧光粉 结构遭到破坏进而影响材料的发光性能。

层状复合型苂光陶瓷是指把黄色或绿色荧光粉 以及红色苂光粉分别堆积成不同的层, 从而制备出 双层或多层复相苂光陶瓷。例如, Bicanic 等 ${ }^{[32]}$ 采用 YAG: $\mathrm{Ce}^{3+}$ 和 $\mathrm{K}_{2} \mathrm{TiF}_{6}: \mathrm{Mn}^{4+}$ 制备了双层复相苂光陶瓷, 并建立了多苂光粉颜色校正分析模型，计算材料的散 射和发光饱和。Yi 等 ${ }^{[50]}$ 采用 YAG:Ce 和 $\mathrm{YAG}: \mathrm{Ce} / \mathrm{Cr}$
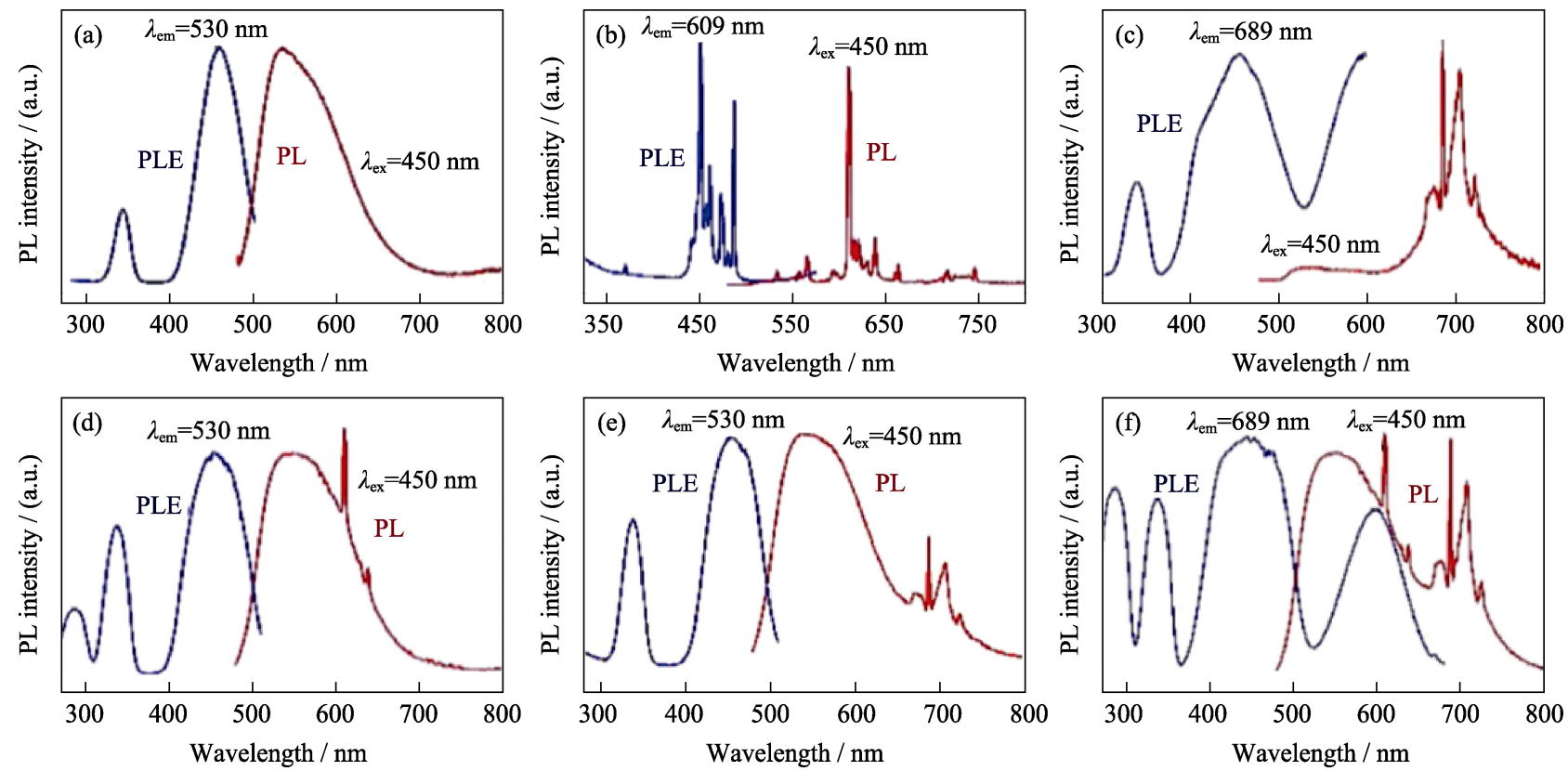

图 $4 \mathrm{YAG:} \mathrm{Ce}^{3+} / \mathrm{Pr}^{3+} / \mathrm{Cr}^{3+}$ 苂光陶瓷的激发和发射光谱 ${ }^{[29]}$

Fig. 4 PL and PLE spectra of YAG: $\mathrm{Ce}^{3+} / \mathrm{Pr}^{3+} / \mathrm{Cr}^{3+}$ phosphor ceramics ${ }^{[2]}$ (a) YAG:Ce; (b) YAG:Pr; (c) YAG:Cr; (d) YAG:Ce,Pr; (e) YAG:Ce,Cr; (f) YAG:Ce,Pr,Cr 
制备出双层复相荧光陶瓷, 获得了相关色温 $4905 \mathrm{~K}$ 、 流明效率 $76 \mathrm{~lm} / \mathrm{W}$ 的照明光源。该方法的局限性在 于红色荧光材料往往会再吸收绿/黄荧光材料发射 的绿光或者黄光, 造成流明效率下降。

薄膜复合型苂光陶瓷是指以黄色或者绿色荧光 陶瓷为祄底, 在上面再制备一层红色荧光薄膜(包括 Phosphor in Silicone (PiS)、Phosphor in Glass (PiG)、

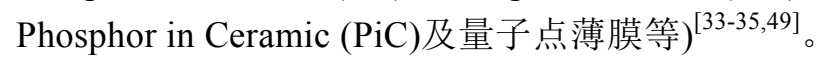
例如, Park 等 ${ }^{[33]}$ 通过在 LuAG:Ce 绿色苂光陶瓷表面 复合二维 $\mathrm{SiN}_{x}$ 光子晶体层以及 $(\mathrm{Sr}, \mathrm{Ca}) \mathrm{AlSiN}_{3}: \mathrm{Eu}$ 红 色 PiS 薄膜制备出复合苂光陶瓷; 随着薄膜中红粉 含量的增加, 样品的流明效率逐渐下降, 相关色温 降低, 显色指数和 R9 显著提高; 其中, 流明效率的 下降是由于随着红光比例的增加, 人眼最为敏感的 绿光比例降低所致; 当薄膜中红粉质量百分比含量 为 $7.5 \%$ 时, 封装的白光 LED 器件显色指数、相关色 温和流明效率分别为 $94 、 4450 \mathrm{~K}$ 和 $71.1 \mathrm{~lm} / \mathrm{W}$ 。 Pricha 等 ${ }^{[34]}$ 通过在 YAG: $\mathrm{Ce}$ 黄色荧光陶瓷表面制备 一层约 $25 \mu \mathrm{m}$ 的 $\mathrm{CaAlSiN}_{3}$ : $\mathrm{Eu}$ 红色 $\mathrm{PiC}$ 薄膜得到相
应的复合荧光陶瓷; 但由于 $\mathrm{Si}-\mathrm{N}$ 和 $\mathrm{Si}-\mathrm{O}$ 键的键能 不同, 以及不同浓度梯度引起的元素扩散, 这导致 两界面处容易发生化学反应，严重影响了复合陶瓷 的发光性能; 为此, 他们通过等离子增强化学气相 沉积(PECVD)在 YAG 苂光陶瓷表面先制备一层 $\mathrm{SiN}_{x}$ 薄膜作为表面钝化层, 然后再利用丝网印刷工 艺涂敷一层 $\mathrm{CaAlSiN}_{3}$ 薄膜, 最后高温烧结得到复合 荧光陶瓷。该工艺在一定程度上抑制了两者之间的 界面反应，增强了复合荧光陶瓷的发光性能。

综上, 石榴石体系苂光陶瓷降低相关色温和提 高显色指数的三种主要方法分别是: 调整基质化学 组成、调整发光中心种类和复合红色苂光材料。表 2 汇总了三种方法的相关研究进展。对于单纯的 YAG:Ce 苂光陶瓷，尽管不同文献资料报道的实验 结果不完全相同, 但总体差异不大, 其发射峰位约 为 $540 \mathrm{~nm}$, 相关色温约为 $5994 \mathrm{~K}$, 显色指数约为 $54.2^{[38-39]}$ 。由表 2 可以看出, 相比于传统 YAG:Ce 苂光陶瓷，采用上述三种方法优化的石榴石体系苂 光陶瓷性能明显改善，几乎全部实现了发射光谱

表 2 石榴石型荧光陶瓷提高显色指数及降低相关色温的三种方法研究进展汇总表

Table 2 Summary of three methods for improving CRI and reducing CCT of garnet type phosphor ceramics

\begin{tabular}{|c|c|c|c|c|c|}
\hline Methods & Composition & Emission peak position/nm & $\mathrm{CCT} / \mathrm{K}$ & CRI & Ref. \\
\hline \multirow{12}{*}{$\begin{array}{l}\text { Adjust matrix } \\
\text { chemical composition }\end{array}$} & GdYAG:Ce & $525-554$ & $2968-4299$ & 64.8 & {$[40]$} \\
\hline & GdYAG:Ce & $528-550$ & $3688-4782$ & 67.1 & {$[51]$} \\
\hline & $\mathrm{Al}_{2} \mathrm{O}_{3}-\mathrm{GdYAG}: \mathrm{Ce}$ & $550^{*}$ & 5010 & 71.4 & {$[52]$} \\
\hline & $\mathrm{MgAl}_{2} \mathrm{O}_{4}-\mathrm{GdYAG}: \mathrm{Ce}$ & $550^{*}$ & 4543 & 70 & {$[18]$} \\
\hline & TbAG:Ce & $556-564$ & $4000-4900$ & - & {$[45]$} \\
\hline & $\mathrm{Al}_{2} \mathrm{O}_{3}-\mathrm{TbAG}: \mathrm{Ce}$ & 555 & 3580 & 63 & {$[53]$} \\
\hline & TGAG:Ce & $550-570$ & 3681 & 74.7 & {$[25]$} \\
\hline & GAGG:Ce & $568-574$ & 3000 & 78.9 & {$[26]$} \\
\hline & GAGG:Ce & 570 & 2800 & 58.7 & {$[47]$} \\
\hline & YMASG:Ce & $537-577$ & 4384 & 81 & {$[27]$} \\
\hline & YMASG:Ce & $533-598$ & $2018-4516$ & - & {$[44]$} \\
\hline & $\mathrm{Al}_{2} \mathrm{O}_{3}$-YMASG:Ce & $552-610$ & 4860 & 82.5 & {$[54]$} \\
\hline \multirow{7}{*}{$\begin{array}{l}\text { Adjust the } \\
\text { luminescence center }\end{array}$} & YAG: $\mathrm{Ce}^{3+} / \mathrm{Pr}^{3+}$ & $535,564,609,637$ & - & 66.9 & {$[30]$} \\
\hline & YAG: $\mathrm{Ce}^{3+} / \mathrm{Cr}^{3+}$ & $534,677,688$, etc. & - & 72 & [31] \\
\hline & YAG: $\mathrm{Ce}^{3+} / \mathrm{Cr}^{3+}$ & $530,690,705$ & 4329 & - & {$[50]$} \\
\hline & YAG: $\mathrm{Ce}^{3+} / \mathrm{Pr}^{3+} / \mathrm{Cr}^{3+}$ & $530,609,689$, etc. & - & 78 & [29] \\
\hline & YAG: $\mathrm{Ce}^{3+} / \mathrm{Mn}^{2+}$ & $520-590$ & $3870-5196$ & 82.5 & [28] \\
\hline & YAG: $\mathrm{Ce}^{3+} / \mathrm{Dy}^{3+}$ & 496,582 , etc. & 5609 & - & {$[55]$} \\
\hline & LuAG:Dy ${ }^{3+}$ & $482,583,675$, etc. & $3485-3619$ & - & {$[56]$} \\
\hline \multirow{4}{*}{$\begin{array}{l}\text { Composite red } \\
\text { fluorescent material }\end{array}$} & LuAG:Ce/(Sr,Ca)AlSiN $3: E u$ & 515,640 & 4450 & 94 & [33] \\
\hline & LuAG:Ce/Eu-doped nitride & $565-587$ & 5800 & 89.4 & [48] \\
\hline & YAG:Ce/ $\mathrm{Sr}_{2} \mathrm{Si}_{5} \mathrm{~N}_{8}: \mathrm{Eu}$ & $610^{*}$ & 3952 & 82 & {$[35]$} \\
\hline & $\mathrm{Al}_{2} \mathrm{O}_{3}$-YAG:Ce/red QD & 552,634 & $3161-6035$ & 80 & [49] \\
\hline
\end{tabular}

Note: the data with "*” refers to the value that can be roughly obtained from the PL spectrum without giving the emission peak position directly in the original text; the cell with "-" means that the relevant data cannot be found in the original text 
红移、相关色温降低和显色指数提高。

\section{2 氮(氧)化物荧光陶瓷}

氮(氧)化物苂光材料兼具颜色丰富、光谱可调、 转化效率高和热猝灭小等优点, 成为目前最具发展 前景的苂光材料之一 ${ }^{[57]}$ 。一方面，氮(氧)化物荧光 材料晶体结构的多样性使其发光颜色涵盖从蓝色到 红色整个可见光范围; 另一方面, 由于氮和氧相比 具有更小的电负性、更大的形式电荷和更高的极化 率, 与氧化物相比, 氮(氧)化物的共价键性更强, 电 子云膨胀和晶体场䢃裂效应显著增强, $5 \mathrm{~d}$ 能级激发 能量降低, 导致光谱红移, 从而使得氮(氧)化物比 氧化物更适合制备性能优异的红色荧光材料 ${ }^{[57]}$ 。相 比于石榴石体系氧化物荧光陶瓷, 氮(氧)化物荧光 陶瓷的研究深度和广度均相对薄弱, 主要原因在于 氮(氧)化物陶瓷具有低扩散系数、高饱和蒸气压和 在高温下容易发生化学反应等缺点使其难以烧结致 密, 而且装备要求高、制备工艺复杂、技术难度高 ${ }^{[8]}$ 。 截止目前, 有关的氮(氧)化物苂光陶瓷材料体系主要 包括: $\mathrm{CaAlSiN}_{3}: \mathrm{Eu}^{2+}$ 和 $\mathrm{M}_{2} \mathrm{Si}_{5} \mathrm{~N}_{8}: \mathrm{Eu}^{2+}(\mathrm{M}=\mathrm{Ca}, \mathrm{Sr}, \mathrm{Ba})$ 红色苂光陶瓷, $\alpha$-Sialon: $\mathrm{Eu}^{2+}$ 黄色荧光陶瓷, $\beta$-Sialon: $\mathrm{Eu}^{2+}$ 和 $\mathrm{MgAlON}: \mathrm{Mn}^{2+}$ 绿色荧光陶瓷, $\mathrm{MgAlON}: \mathrm{Ce}^{3+}$ 蓝色苂光陶瓷以及 $\mathrm{AlN}: \mathrm{Ce}^{3+}$ 白色苂光 陶瓷等 ${ }^{[58-65]}$ 。相关研究进展分述如下。

$\mathrm{M}_{2} \mathrm{Si}_{5} \mathrm{~N}_{8}: \mathrm{Eu}^{2+}(\mathrm{M}=\mathrm{Ca}, \mathrm{Sr}, \mathrm{Ba})$ 是一种性能优异的 代表性氮化物红色苂光粉 ${ }^{[66]}$ 。针对汽车大灯等大功 率固态照明应用需求，荷兰飞利浦(Philips Lumileds) 和德国欧司朗(Osram)等先后制备了 $\mathrm{M}_{2} \mathrm{Si}_{5} \mathrm{~N}_{8}$ : $\mathrm{Eu}^{2+}$
$(\mathrm{M}=\mathrm{Ca}, \mathrm{Sr}, \mathrm{Ba})$ 红色苂光陶瓷并和 LED 芯片封装成照 明器件 ${ }^{[63,65]}$ 。 $\mathrm{CaAlSiN}_{3}: \mathrm{Eu}^{2+}$ 是另一种代表性的氮化物 红色苂光粉 ${ }^{[66]}$ 。与 $\mathrm{Sr}_{2} \mathrm{Si}_{5} \mathrm{~N}_{8}: \mathrm{Eu}^{2+}$ 相比, $\mathrm{CaAlSiN}_{3}: \mathrm{Eu}^{2+}$ 在热稳定性、量子效率等方面具有独特优势, 不仅 可广泛应用于高显色指数白光 LED 照明光源, 还可 应用于广色域白光 LED 背光源 ${ }^{[58]}$ 。2015 年, 德国 西门子公司 Pricha 等 ${ }^{[62]}$ 率先研究了 $\mathrm{CaAlSiN}_{3}: \mathrm{Eu}^{2+}$ 荧光陶瓷的烧结，但是其致密度仅为 $80 \%$ 。2016 年, 本研究团队巧妙地采用 $\mathrm{Si}_{3} \mathrm{~N}_{4}$ 和 $\mathrm{SiO}_{2}$ 作为双助熔剂, 通过放电等离子体烧结（SPS）技术制备了半透明 $\mathrm{CaAlSiN}_{3}: \mathrm{Eu}^{2+}$ 红色苂光陶瓷 ${ }^{[6,58]}$; 如图 5 所示, $\mathrm{Si}_{3} \mathrm{~N}_{4}$ 和 $\mathrm{SiO}_{2}$ 可与 $\mathrm{CaAlSiN}_{3}$ 发生反应生成 $\mathrm{CaAlSiN}_{3}-\mathrm{Si}_{2} \mathrm{~N}_{2} \mathrm{O}$ 固溶体, 不会对发光性能造成破坏, 由于 $\mathrm{SiO}_{2}$ 熔点 较低, 可在较低温度下熔化生成液相促进致密化烧 结。研究表明, 具有核 $\left(\mathrm{CaAlSiN}_{3}\right)$-壳 $\left(\mathrm{CaAlSiN}_{3}-\mathrm{Si}_{2} \mathrm{~N}_{2} \mathrm{O}\right)$ 结构的红色发光苂光颗粒均匀弥散在新生成的不发 光 $\mathrm{Ca}-\alpha$-sialon 基质中, 得到的苂光陶瓷具有优异的 热稳定性(热猝灭性能较荧光粉提高 15\%)和热导率 $\left(4 \mathrm{~W} \cdot \mathrm{m}^{-1} \cdot \mathrm{K}^{-1}\right)$, 在 $450 \mathrm{~nm}$ 激发光下其外量子效率达 $60 \%$ (是粉体的 $87 \%$ )。在蓝色辐照激光的功率密度 为 $1.5 \mathrm{~W} / \mathrm{mm}^{2}$ 时, 开始发生发光饱和现象, 此时的 光通量和流明效率分别为 $200 \mathrm{~lm}$ 和 $42.2 \mathrm{~lm} / \mathrm{W}$ 。

$\alpha$-Sialon: $\mathrm{Eu}^{2+}$ 黄色荧光粉和 $\beta$-Sialon: $\mathrm{Eu}^{2+}$ 绿色 荧光粉也是性能优异的两种代表性氮(氧)化物荧光 粉 ${ }^{[67-68]}$ 。 $\mathrm{Eu}^{2+}$ 的离子半径较大, 很难得到单一的 $\alpha$-Sialon 相, 需要添加一些较小的阳离子(如 $\mathrm{Mg}^{2+}$ 、 $\mathrm{Ca}^{2+} 、 \mathrm{Li}^{+} 、 \mathrm{Y}^{3+}$ 或一些稀土离子等)作为稳定剂 ${ }^{[60]}$ 。
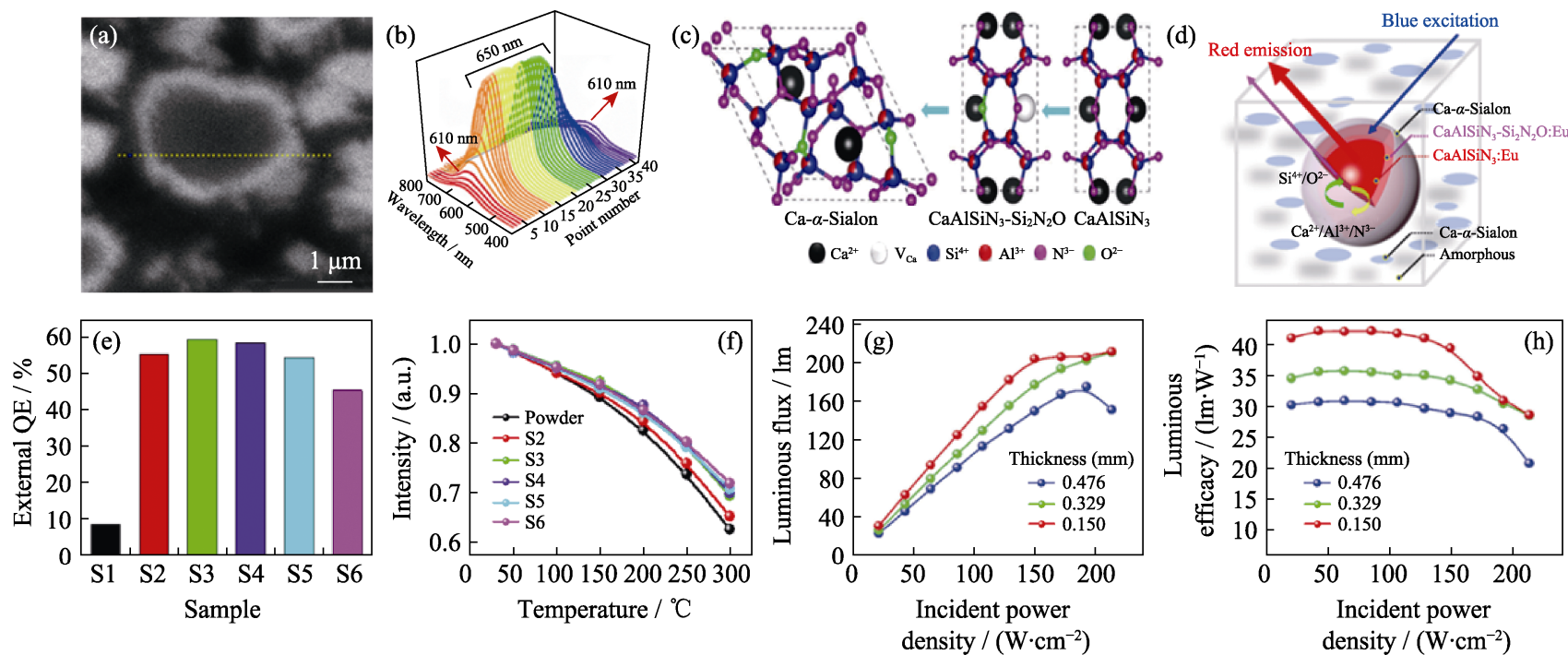

图 $5 \mathrm{CaAlSiN}_{3}: \mathrm{Eu}^{2+}$ 苂光陶瓷 ${ }^{[6,58]}$

Fig. $5 \mathrm{CaAlSiN}_{3}: \mathrm{Eu}^{2+}$ phosphor ceramics ${ }^{[6,58]}$

(a) Single $\mathrm{CaAlSiN}_{3}: \mathrm{Eu}^{2+}$ grain CL spectral line scan; (b) CL spectra; (c) Crystal structure transition; (d) Core-shell structure schematic diagram; (e) Quantum efficiency of samples; (f) Thermal stability of samples with different $\mathrm{Si}_{3} \mathrm{~N}_{4}$ and $\mathrm{SiO}_{2}$ contents;

(g) Influence of incident power density on luminous flux; (h) Luminous efficiency of samples

Colorful figures are available on website 
韩国鲜文大学 Joshi 等 ${ }^{[59-60]}$ 开展了 Sialon 苂光陶瓷的 深入研究; 采用热压烧结法制备出 $\mathrm{Mg}-\alpha$-Sialon: $\mathrm{Eu}$ 透 明黄色苂光陶瓷，在 350 450 nm 紫外-蓝光激发下的 发射波长为 $570 \mathrm{~nm}$, 流明效率最高可达 $88.59 \mathrm{~lm} / \mathrm{W}^{[60]}$; 此外还研究了 $\mathrm{Eu}^{2+} / \mathrm{Gd}^{3+} / \mathrm{Pr}^{3+}$ 掺杂及共掺杂 Sialon 苂 光陶瓷，通过 $\mathrm{Gd}^{3+} / \mathrm{Eu}^{2+}$ 共掺杂可提高 $\alpha$-Sialon 相含 量, 并提高荧光陶瓷的透明度, 通过 $\mathrm{Pr}^{3+} / \mathrm{Eu}^{2+}$ 共掺 杂则使发射光谱在红色波段显示出肩峰; 所制备的 $\beta$-Sialon:Eu 绿色荧光陶瓷在蓝光激发下发射 $552 \mathrm{~nm}$ 的绿光, 流明效率最高达 $58.78 \mathrm{~lm} / \mathrm{W}$, 相关色温为 $5097 \mathrm{~K}^{[59]}$ 。

被研究的氮(氧)化物苂光陶瓷还有 $\mathrm{MgAlON}: \mathrm{Ce}^{3+}$ 蓝色荧光陶瓷、 $\mathrm{MgAlON}: \mathrm{Mn}^{2+}$ 绿色苂光陶瓷和 $\mathrm{AlN}: \mathrm{Ce}^{3+}$ 白色苂光陶瓷。 $\mathrm{Liu}$ 等 ${ }^{\left[{ }^{[36}\right]}$ 采用无压烧结制 备了 $\mathrm{MgAlON}: \mathrm{Ce}^{3+}$ 蓝色苂光陶瓷, 在 $320 \mathrm{~nm}$ 的紫 外光激发下, 苂光陶瓷发射 $410 \mathrm{~nm}$ 的蓝光; 当 $\mathrm{Ce}^{3+}$ 原子掺杂浓度为 $0.01 \%$ 时, 苂光陶瓷保持较高的透 过率, 维氏硬度 $13.7 \mathrm{GPa}$, 内量子效率 $42 \%$; 当 $\mathrm{Ce}^{3+}$ 原子掺杂浓度高于 $0.01 \%$ 时, 因形成第二相而 严重影响苂光陶瓷的透过率、机械性能和热稳定性。 $\mathrm{Li}$ 等 $^{[61]}$ 采用无压烧结结合热等静压处理(HIP)制备 出 MgAlON: $\mathrm{Mn}^{2+}$ 绿色苂光陶瓷, 研究表明 $\mathrm{Mn}^{2+}$ 占 据了 $\mathrm{MgAlON}$ 尖晶石晶格的四面体位置; 由于 $\mathrm{Mn}^{2+}$ 的 ${ }^{4} \mathrm{~T}_{1} \rightarrow{ }^{6} \mathrm{~A}_{1}$ 能级跃迁, 苂光陶瓷在 $445 \mathrm{~nm}$ 蓝光 激发下发射出 $513 \mathrm{~nm}$ 绿光, 内量子效率 $47 \%$, 具有 良好的热稳定性。Wieg 等 ${ }^{[64]}$ 采用电流活化压力辅助 致密化(CAPAD)方法制备出 $\mathrm{AlN}: \mathrm{Ce}^{3+}$ 白色荧光陶瓷, 在 $375 \mathrm{~nm}$ 紫外光激发下其发射光谱覆盖了几乎整 个可见光范围, 因此不需颜色混合即可直接获得 白光; 光致发光是源于 $\mathrm{AlN}$ 的本征缺陷及 $\mathrm{Ce}^{3+}$ 的 电子跃迁, 可通过改变 $\mathrm{Ce}^{3+}$ 浓度和工艺参数(如 CAPAD 温度)来调节发光强度; 样品的最佳发光非 常接近太阳光, 色坐标为 $(x=0.3547, y=0.3810)$, 相 关色温为 $4776 \mathrm{~K}$ 。

\section{3 未来发展方向}

针对大功率固态照明的应用需求, 尚需进一步 提高苂光陶瓷材料的热稳定性、光提取效率以及显 色指数等性能, 基于此, 本综述对大功率固态照明用 苂光陶瓷材料未来的发展方向进行如下几点展望。

\section{1 提高光提取效率}

不同于普通 LED 照明, 激光是一种高度聚焦的 点光源, 在激光照明中需要通过提高光提取效率来 解决“黄环效应”(Yellow Ring Effect)。本文已经详细
分析了提高大功率固态照明用苂光陶瓷光提取效率 的几种方法, 主要包括引入不发光第二相、引入气 孔、表面粗粘化和表面修饰等。其中, 最具有普适 性的方法是通过引入不发光第二相来改变激发光在 材料中的传播路径, 从而提高苂光陶瓷的光提取效 率。但需要指出的是, 对于透射模式的封装器件, 为 保证复相荧光陶瓷具有较高的透过率, 所引入的第 二相应为透明或半透明陶瓷, 在蓝光波段应具有高 透过率(即几乎不吸收蓝光), 且要与苂光粉的折射 率相匹配。对于最常用的 YAG:Ce 苂光陶瓷 $(n=1.84)$ 搭配蓝光 LED 芯片的封装模式, 按照上述原则可选 择的第二相材料包含在图 6 中的阴影区域内, 主要 包括 $\mathrm{AlN} 、 \mathrm{Y}_{2} \mathrm{O}_{3} 、 \mathrm{Gd}_{2} \mathrm{O}_{3} 、 \mathrm{Al}_{2} \mathrm{O}_{3} 、 \mathrm{MgO}$ 和羟基磷灰 石 ${ }^{[17]}$ 。同时, 提高光提取效率必将增加产热, 这在 一定程度上会影响热稳定性, 因此需要平衡好二者 之间的关系。

\section{2 提高显色指数}

由于缺少红光成分, 目前大功率固态照明用苂 光陶瓷材料的显色指数普遍较低。本文分析讨论了 提高大功率固态照明用荧光陶瓷显色指数的三种方 法, 分别是调整基质化学组成、调整发光中心种类 和复合红色苂光材料等。尽管这些方法可在一定程 度上提高苂光陶瓷的显色指数, 但前两种方法补充 的红光成分有限, 并且通过调整基质化学组成或发 光中心必然引起不同程度的晶格畸变, 往往会引起 流明效率和透明度一定程度降低; 而对于复合红色 荧光材料而言, 引入的红色荧光材料通常会再吸 收绿/黄色苂光材料发射的绿/黄光, 也会造成流明 效率的降低。因此, 设计、开发性能优异的新型红 色荧光陶瓷至关重要。但截止目前, 已报道的红色 荧光陶瓷种类非常有限, 主要有 $\mathrm{Eu}^{2+}$ 掺杂氮化物 苂光陶瓷和 $\mathrm{Mn}^{4+}$ 掺杂石榴石体系苂光陶瓷两类, 其 性能都有待进一步提高。相比于 $\mathrm{LuAG}: \mathrm{Mn}^{4+}$

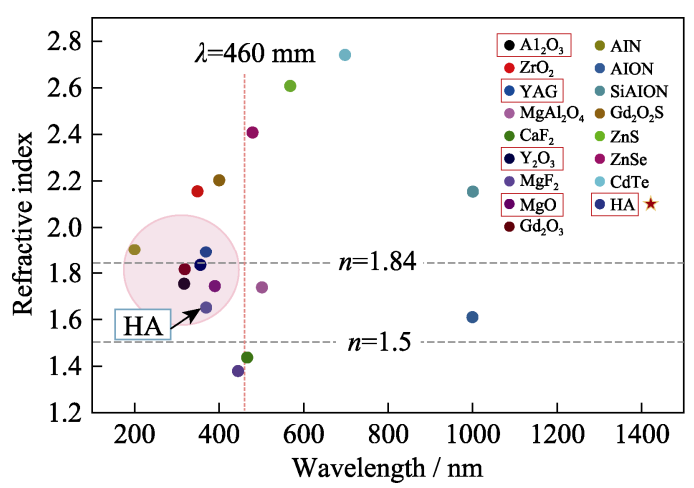

图 6 常见透明陶瓷的透光区域和折射率 ${ }^{[17]}$

Fig. 6 Light-transmitting area and refractive index of common transparent ceramics ${ }^{[17]}$

Colorful figures are available on website 
红色荧光陶瓷, $\mathrm{CaAlSiN}_{3}: \mathrm{Eu}^{2+}$ 红色荧光陶瓷的性能 更加优异, 但氮化物低扩散系数和高饱和蒸气压的 特性使高致密氮化物荧光陶瓷的制备非常困难。因 此, 制备性能优异的大功率固态照明用红色苂光陶 瓷也必将是未来一段时期的研究重点和难点。

\section{3 采用新型荧光粉材料制备荧光陶瓷}

截止目前, 研究人员已经对以 YAG:Ce 为代表 的石榴石体系荧光陶瓷开展了大量卓有成效的研究, 部分成果已转入半商业化应用阶段, 大规模商业化 生产和应用指日可待。然而，受制于 YAG:Ce 基苂 光陶瓷难以克服的低显色指数等不足, 尚难以满足 高端照明的商业应用需求, 因此, 研发或采用性能 优异的新型荧光粉材料制备性能优异的荧光陶瓷必 将是未来大功率固态照明发展的重要方向之一。20 世纪末以来, 科研人员已经开发出成百上千种新型 荧光粉, 从中优选或研发新的荧光粉材料以制备荧 光陶瓷是一种重要思路。从遴选原则来看, 具有高 量子效率、小的热猝灭性、尤其是属于立方晶系的 苂光粉材料体系应成为关注的重点, 其中高量子效 率和小的热猝灭性有利于提高荧光陶瓷的光学性能, 而立方晶系材料更容易实现透明或半透明的结构。 例如, Zhang 等 ${ }^{[69]}$ 采用高温固相反应烧结制备了 $\mathrm{MgAl}_{2} \mathrm{O}_{4}: \mathrm{Mn}^{4+}$ 尖晶石体系红色透明苂光陶瓷。虽然 此前有将 $\mathrm{MgAl}_{2} \mathrm{O}_{4}$ 作为第二相引入 $\mathrm{YAG}$ 基荧光粉 制备复相荧光陶瓷的研究, 但还没有尖晶石体系单 相荧光陶瓷的研究报道 ${ }^{[18]} 。 \mathrm{MgAl}_{2} \mathrm{O}_{4}$ 和 $\mathrm{Y}_{3} \mathrm{Al}_{5} \mathrm{O}_{12}$ 同 为立方晶系，均可烧结为透明陶瓷，因此 $\mathrm{MgAl}_{2} \mathrm{O}_{4}$ 体系荧光陶瓷有望像石榴石体系苂光陶瓷一样获得 更加广泛的重视和研究。近期, Arredondo 等 ${ }^{[70]}$ 制备 了一种新型 $\mathrm{Sr}_{2.53} \mathrm{Ba}_{0.47} \mathrm{AlO}_{4} \mathrm{~F}: \mathrm{Ce}^{3+}$ 氧氟化物荧光陶瓷, 这暗示了其他氟化物或氧氟化物荧光粉在大功率固 态照明用荧光陶瓷领域的潜在应用前景。此外, 采 用纳米级苂光粉或第二相, 特别是采用液相法合成 的具有特殊显微结构的纳米级荧光粉或第二相所制备 的荧光陶瓷，在光学和机械性能方面往往优于传统的 采用微米级苂光粉或第二相制备的荧光陶瓷 ${ }^{[12,17,39]}$ 。

\section{4 采用新型技术工艺制备荧光陶瓷}

苂光陶瓷的制备工艺过程主要包括混料、过篮、 干压成型、冷等静压、脱粘、烧结和加工，也有少 量通过制备浆料、流延成型等工艺制备荧光陶瓷的 研究报道, 这些均属于陶瓷材料的传统制备工艺。 随着材料制备新技术、新工艺的不断发展, 采用先 进的新技术、新工艺制备大功率固态照明用苂光陶 瓷也是一个可行的研究思路。例如, 近期 $\mathrm{Hu}$ 等 ${ }^{[71]}$ 首次尝试将 3D 打印增材制造技术用于苂光陶瓷的
制备, 制备出亚毫米级圆柱形 $\mathrm{Al}_{2} \mathrm{O}_{3}-\mathrm{YAG}: \mathrm{Ce}$ 复相 荧光陶瓷, 其饱和功率密度 $\left(20.7 \mathrm{~W} / \mathrm{mm}^{2}\right)$ 优于采用 传统工艺制备的样品; 借助 3D 打印新工艺可以通 过设计和调控苂光陶瓷的显微结构使其获得优异的 发光性能，具有快速、高效、低成本、高精度控制 等优点, 是制备大功率固态照明用苂光陶瓷的未来 发展方向之一。

\section{5 加强复相苂光陶瓷的研究}

与 YAG:Ce 单相荧光陶瓷相比, $\mathrm{Al}_{2} \mathrm{O}_{3}-\mathrm{YAG}: \mathrm{Ce}$ 复相苂光陶瓷具有更小的热猝灭性、更高的发光饱 和阈值和流明效率, 这是由于引入 $\mathrm{Al}_{2} \mathrm{O}_{3}$ 显著提高 了苂光陶瓷的热导率从而增强了散热, 还可以作为 散射中心提高光提取效率 ${ }^{[9,11]}$ 。针对激光照明的应 用需求，引入第二相显得更为重要。激光是典型的 高方向性的点光源, 光斑面积小, 光线直接穿过单 相透明陶瓷会导致光提取效率较低, 而第二相作为 散射中心可以改变光线的传播路径从而提高光一致 性、光提取效率和流明效率。激光又是高能量光源, 激光照射的样品表面可以达到 $200{ }^{\circ} \mathrm{C}$ 以上, 这需要 样品具有良好的热导率来增强散热, 而引入高热导 第二相可以在很大程度上缓解这个问题，从而提高 器件的热稳定性和发光饱和阈值。目前, 已报道的 荧光陶瓷的第二相局限于 $\mathrm{Al}_{2} \mathrm{O}_{3} 、 \mathrm{MgAl}_{2} \mathrm{O}_{4} 、 \mathrm{CaF}_{2}$ 、 $\mathrm{HA}$ 等少数化合物, 因此探索更合适的第二相来制 备复相苂光陶瓷将成为未来大功率固态照明用荧光 陶瓷另外一个重要的发展方向。

\section{6 加强激光照明透射模式荧光陶瓷的研究}

针对激光照明产生热量高的特点, 激光照明多 采用远程封装, 主要包括反射模式和透射模式两种 类型。目前报道的研究多采用反射模式, 这是由于 反射模式对荧光陶瓷的透明度要求相对较低, 且苂 光陶瓷可以粘贴在高导热基板上来增强散热效果。 但反射模式存在封装结构复杂、发光器件的体积和 结构较大、光学性能的可调性难以满足不同应用场 合需求等缺点 ${ }^{[11,17]}$ 。相比之下, 透射模式对于发光器 件光学性能的裁剪设计更加简便, 未来发展的潜力巨 大。近期, Huang 等 ${ }^{[17]}$ 采用所制备的 HA-YAG:Ce 复 相苂光陶瓷，通过透射封装模式获得了流明效率 $170 \mathrm{~lm} / \mathrm{W}$ 、相关色温低于 $4500 \mathrm{~K}$ 且色纯度高达 $90 \%$ 的 WLED 器件。

\section{7 加强模拟计算和实验相结合的研究}

现有的研究报道基本都是实验研究的结果, 将 模拟计算和实验研究有机结合也将是未来的发展趋 势之一。通过模拟计算获得大功率固态照明器件的 最大辐射通量、发光饱和阈值、光提取效率和一致 
性、色度坐标、相关色温、显色指数等技术参数是 推动大功率固态照明未来快速发展的重要途径。同 时, 模拟计算还将为遴选合适的发光材料及其组合 以及材料设计提供重要指导。此外, 开展实验结果 与模拟计算的比较研究, 也将有助于更加深入地揭 示其它未知因素及其影响规律 ${ }^{[3]}$ 。例如, Bicanic 等 ${ }^{[32]}$ 针对苂光材料的散射和发光饱和问题设计开发了一 个多苂光粉颜色校正分析的苂光层模型 (Color Correction Analysis for Multiple Phosphors), 采用该 模型预测并证实了通过 YAG: $\mathrm{Ce}^{3+}$ 和 $\mathrm{K}_{2} \mathrm{TiF}_{6}: \mathrm{Mn}^{4+}$ 两 种荧光粉均匀混合制备出的荧光陶瓷要比层状复合 结构的苂光陶瓷性能更佳, 并优化了两种苂光粉的 配比, 这在很大程度上克服了 $\mathrm{K}_{2} \mathrm{TiF}_{6}: \mathrm{Mn}^{4+}$ 苂光粉 因苂光寿命长 $(4.8 \mathrm{~ms})$ 容易产生发光饱和的缺点。

\section{4 结束语}

本文探讨了大功率固态照明用苂光陶瓷的设计 原则, 介绍了固态照明用荧光陶瓷的材料体系及其 研究进展, 并展望了未来的发展方向。苂光陶瓷在 综合性能方面优于苂光玻璃、苂光薄膜、荧光单晶 以及量子阱, 被认为是综合性能最优、最有发展前 景的大功率固态照明用苂光材料。未来, 苂光陶瓷 材料将在汽车大灯、户外照明、激光电视、激光影 院、可见光通信、激光雷达等领域得到更广泛的应 用和发展。期望通过本文的报道和总结, 可以吸引 更多的科研人员投入到苂光陶瓷的研究之中, 为大 功率固态照明的蓬勃发展贡献力量。

\section{参考文献:}

[1] SCHUBERT E F, KIM J K. Solid-state light sources getting smart. Science, 2005, 308(5726): 1274-1278.

[2] WIERER J J, TSAO J Y, SIZOV D S. Comparison between blue lasers and light-emitting diodes for future solid-state lighting. Laser \& Photonics Reviews, 2013, 7(6): 963-993.

[3] LI S, WANG L, HIROSAKI N, et al. Color conversion materials for high-brightness laser-driven solid-state lighting. Laser \& Photonics Reviews, 2018, 12(12): 1800173.

[4] FAN F, TURKDOGAN S, LIU Z, et al. A monolithic white laser. Nat. Nanotechnol., 2015, 10(9): 796-803.

[5] YAO Q, HU P, SUN P, et al. YAG: $\mathrm{Ce}^{3+}$ transparent ceramic phosphors brighten the next-generation laser-driven lighting. $A d v$. Mater., 2020, 32(19): 1907888.

[6] LI S X, TANG D M, TIAN Z F, et al. New insights into the microstructure of translucent $\mathrm{CaAlSiN}_{3}: \mathrm{Eu}^{2+}$ phosphor ceramics for solid-state laser lighting. Journal of Materials Chemistry C, 2017, 5(5): 1042-1051.

[7] LENEF A, KELSO J, ZHENG Y, et al. Radiance limits of ceramic phosphors under high excitation fluxes. Proceedings of SPIE, 2013, 8841: 884107.

[8] XU Y R, LI S X, ZHENG P, et al. A search for extra-high brightness laser-driven color converters by investigating thermally-induced luminance saturation. Journal of Materials Chemistry C, 2019, 7(37): 11449-11456.
[9] COZZAN C, LHEUREUX G, O'DEA N, et al. Stable, heat-conducting phosphor composites for high-power laser lighting. ACS Appl. Mater. Interfaces, 2018, 10(6): 5673-5681.

[10] LI S, ZHU Q, TANG D, et al. $\mathrm{Al}_{2} \mathrm{O}_{3}$-YAG:Ce composite phosphor ceramic: a thermally robust and efficient color converter for solid state laser lighting. Journal of Materials Chemistry C, 2016, 4(37): 8648-8654.

[11] WANG J C, TANG X Y, ZHENG P, et al. Thermally self-managing YAG:Ce- $\mathrm{Al}_{2} \mathrm{O}_{3}$ color converters enabling high-brightness laser-driven solid state lighting in a transmissive configuration. Journal of Materials Chemistry C, 2019, 7(13): 3901-3908.

[12] MA X G, LI X Y, LI J Q, et al. Pressureless glass crystallization of transparent yttrium aluminum garnet-based nanoceramics. Nature Communications, 2018, 9(1): 1175.

[13] PARK J, KIM J, KWON H. Phosphor-aluminum composite for energy recycling with high-power white lighting. Advanced Optical Materials, 2017, 5(19): 1700347.

[14] ZHENG P, LI S, WEI R, et al. Unique design strategy for laser-driven color converters enabling superhigh-luminance and high-directionality white light. Laser \& Photonics Reviews, 2019, 13(10): 14930-14940.

[15] ZHANG L, SUN B, GU L, et al. Enhanced light extraction of single-surface textured YAG:Ce transparent ceramics for high power white LEDs. Applied Surface Science, 2018, 455: 425-432.

[16] ZHANG Y, HU S, WANG Z, et al. Pore-existing $\mathrm{Lu}_{3} \mathrm{Al}_{5} \mathrm{O}_{12}: \mathrm{Ce}$ ceramic phosphor: an efficient green color converter for laser light source. Journal of Luminescence, 2018, 197: 331-334.

[17] HUANG P, ZHOU B, ZHENG Q, et al. Nano wave plates structuring and index matching in transparent hydroxyapatite-YAG: $\mathrm{Ce}$ composite ceramics for high luminous efficiency white light-emitting diodes. Advanced Materials, 2019, 32(1): 1905951.

[18] LIU X, QIAN X, ZHENG P, et al. Preparation and optical properties of $\mathrm{MgAl}_{2} \mathrm{O}_{4}$-Ce:GdYAG composite ceramic phosphors for white LEDs. Journal of the European Ceramic Society, 2019, 39(15): 4965-4971.

[19] SUN B H, ZHANG L, HUANG G C, et al. Surface texture induced light extraction of novel Ce:YAG ceramic tubes for outdoor lighting. Journal of Materials Science, 2019, 54(1): 159-171.

[20] WAGNER A, RATZKER B, KALABUKHOV S, et al. Enhanced external luminescence quantum efficiency of ceramic phosphors by surface roughening. Journal of Luminescence, 2019, 213: 454-458.

[21] PARK H K, OH J R, DO Y R. 2D SiN ${ }_{x}$ photonic crystal coated $\mathrm{Y}_{3} \mathrm{Al}_{5} \mathrm{O}_{12}: \mathrm{Ce}^{3+}$ ceramic plate phosphor for high-power white light-emitting diodes. Optics Express, 2011, 19(25): 25593-25601.

[22] PARK H K, YOON S W, CHOI D Y, et al. Fabrication of wafer-scale $\mathrm{TiO}_{2}$ nanobowl arrays via a scooping transfer of polystyrene nanospheres and atomic layer deposition for their application in photonic crystals. Journal of Materials Chemistry C, 2013, 1(9): 1732-1738.

[23] TANG Y, ZHOU S, CHEN C, et al. Composite phase ceramic phosphor of $\mathrm{Al}_{2} \mathrm{O}_{3}$-Ce:YAG for high efficiency light emitting. Opt. Express, 2015, 23(14): 17923-17928.

[24] HU S, ZHANG Y, WANG Z, et al. Phase composition, microstructure and luminescent property evolutions in "light-scattering enhanced" $\mathrm{Al}_{2} \mathrm{O}_{3}-\mathrm{Y}_{3} \mathrm{Al}_{5} \mathrm{O}_{12}: \mathrm{Ce}^{3+}$ ceramic phosphors. Journal of the European Ceramic Society, 2018, 38(9): 3268-3278.

[25] CHEN J, TANG Y, YI X, et al. Fabrication of (Tb, Gd $)_{3} \mathrm{Al}_{5} \mathrm{O}_{12}: \mathrm{Ce}^{3+}$ phosphor ceramics for warm white light-emitting diodes application. Optical Materials Express, 2019, 9(8): 3333-3341.

[26] LIU S, SUN P, LIU Y, et al. Warm white light with a high color rendering index from a single $\mathrm{Gd}_{3} \mathrm{Al}_{4} \mathrm{GaO}_{12}: \mathrm{Ce}^{3+}$ transparent ceramic for high-power LEDs and LDs. ACS Appl. Mater. Interfaces, 2018, 11(2): 2130-2139.

[27] TIAN Y, TANG Y, YI X, et al. The analyses of structure and luminescence in $\left(\mathrm{Mg}_{y} \mathrm{Y}_{3-y}\right)\left(\mathrm{Al}_{5-y} \mathrm{Si}_{y}\right) \mathrm{O}_{12}$ and $\mathrm{Y}_{3}\left(\mathrm{Mg}_{x} \mathrm{Al}_{5-2 x} \mathrm{Si}_{x}\right) \mathrm{O}_{12}$ ceramic phosphors. Journal of Alloys and Compounds, 2020, 813: 152236.

[28] AO G, TANG Y, YI X, et al. Red emission generation in $\mathrm{Ce}^{3+} / \mathrm{Mn}^{2+}$ co-doping $\mathrm{Y}_{3} \mathrm{Al}_{5} \mathrm{O}_{12}$ phosphor ceramics for warm white lighting emitting diodes. Journal of Alloys and Compounds, 2019, 798: 695-699

[29] FENG S, QIN H, WU G, et al. Spectrum regulation of YAG:Ce 
transparent ceramics with $\mathrm{Pr}, \mathrm{Cr}$ doping for white light emitting diodes application. Journal of the European Ceramic Society, 2017, 37(10): 3403-3409.

[30] TANG Y, ZHOU S, YI X, et al. The characterization of Ce/Pr-doped YAG phosphor ceramic for the white LEDs. Journal of Alloys and Compounds, 2018, 745: 84-89.

[31] TANG Y R, ZHOU S M, YI X Z, et al. The Cr-doping effect on white light emitting properties of Ce:YAG phosphor ceramics. Journal of the American Ceramic Society, 2017, 100(6): 2590-2595.

[32] BICANIC K T, LI X Y, SABATINI R P, et al. Design of phosphor white light systems for high-power applications. ACS Photonics, 2016, 3(12): 2243-2248.

[33] PARK H K, OH J H, KANG H, et al. Hybrid 2D photonic crystalassisted $\mathrm{Lu}_{3} \mathrm{Al}_{5} \mathrm{O}_{12}$ :Ce ceramic-plate phosphor and free-standing red film phosphor for white LEDs with high color-rendering index. ACS Appl. Mater. Interfaces, 2015, 7(8): 4549-4559.

[34] PRICHA I, ROSSNER W, MOOS R, et al. Layered ceramic phosphors based on $\mathrm{CaAlSiN}_{3}$ : $\mathrm{Eu}$ and YAG:Ce for white lightemitting diodes. Journal of the American Ceramic Society, 2016, 99(1): 211-217.

[35] SONG Y H, HAN G S, JI E K, et al. The novel design of a remote phosphor ceramic plate for white light generation in high power LEDs. Journal of Materials Chemistry C, 2015, 3(24): 6148-6152.

[36] LIU X, CHEN B, TU B, et al. Variation of structure and photoluminescence properties of $\mathrm{Ce}^{3+}$ doped MgAlON transparent ceramics with different doping content. Materials, 2017, 10(7): 792.

[37] JOSHI B, LEE S W. Luminescence properties of $\mathrm{Eu}^{2+}, \mathrm{Gd}^{3+}$ and $\mathrm{Pr}^{3+}$ doped translucent Sialon phosphors. Journal of Rare Earths, 2015, 33(11): 1142-1147.

[38] NISHIURA S, TANABE S, FUJIOKA K, et al. Properties of transparent Ce:YAG ceramic phosphors for white LED. Optical Materials, 2011, 33(5): 688-691.

[39] SONG Y H, JI E K, JEONG B W, et al. High power laser-driven ceramic phosphor plate for outstanding efficient white light conversion in application of automotive lighting. Sci. Rep., 2016, 6: 31206.

[40] HU C, SHI Y, FENG X Q, et al. YAG:Ce/(Gd,Y)AG:Ce duallayered composite structure ceramic phosphors designed for bright white light-emitting diodes with various CCT. Optics Express, 2015, 23(14): 18243-18255.

[41] ANGLE J P, WANG Z J, DAMES C, et al. Comparison of two-phase thermal conductivity models with experiments on dilute ceramic composites. Journal of the American Ceramic Society, 2013, 96(9): 2935-2942.

[42] GU C, WANG X J, XIA C, et al. A new $\mathrm{CaF}_{2}$-YAG: Ce composite phosphor ceramic for high-power and high-color-rendering WLEDs. Journal of Materials Chemistry C, 2019, 7(28): 8569-8574.

[43] LIU Y, HU S, ZHANG Y, et al. Crystal structure evolution and luminescence property of $\mathrm{Ce}^{3+}$-doped $\mathrm{Y}_{2} \mathrm{O}_{3}-\mathrm{Al}_{2} \mathrm{O}_{3}-\mathrm{Sc}_{2} \mathrm{O}_{3}$ ternary ceramics. Journal of the European Ceramic Society, 2020, 40(3): $840-846$.

[44] DU Q P, FENG S W, QIN H M, et al. Massive red-shifting of $\mathrm{Ce}^{3+}$ emission by $\mathrm{Mg}^{2+}$ and $\mathrm{Si}^{4+}$ doping of YAG:Ce transparent ceramic phosphors. Journal of Materials Chemistry C, 2018, 6(45): 12200-12205.

[45] BI J, LI J G, ZHU Q, et al. Yellow-emitting $\left(\mathrm{Tb}_{1-x} \mathrm{Ce}_{x}\right)_{3} \mathrm{Al}_{5} \mathrm{O}_{12}$ phosphor powder and ceramic $(0 \leqslant x \leqslant 0.05)$ : phase evolution, photoluminescence, and the process of energy transfer. Ceramics International, 2017, 43(11): 8163-8170.

[46] JI E K, SONG Y H, BAK S H, et al. The design of a ceramic phosphor plate with functional materials for application in high power LEDs. Journal of Materials Chemistry C, 2015, 3(48): 12390-12393.

[47] LIU Y, LIU S, SUN P, et al. Transparent ceramics enabling high luminous flux and efficacy for the next-generation high-power LED light. ACS Appl. Mater. Interfaces, 2019, 11(24): 21697-21701.

[48] KRASNOSHCHOKA A, THORSETH A, DAM-HANSEN C, et al. Investigation of saturation effects in ceramic phosphors for laser lighting. Materials, 2017, 10(12): 1407.

[49] XU J, HU B F, XU C, et al. A unique color converter geometry for laser-driven white lighting. Optical Materials, 2018, 86: 286-290.

[50] YI X, ZHOU S, CHEN C, et al. Fabrication of Ce:YAG, Ce,Cr:YAG and $\mathrm{Ce}: \mathrm{YAG} / \mathrm{Ce}, \mathrm{Cr}: \mathrm{YAG}$ dual-layered composite phosphor ceramics for the application of white LEDs. Ceramics International, 2014, 40(5): 7043-7047.

[51] LIU X, ZHOU H, HU Z, et al. Transparent Ce:GdYAG ceramic color converters for high-brightness white LEDs and LDs. Optical Materials, 2019, 88: 97-102.

[52] LIU X, QIAN X, HU Z, et al. $\mathrm{Al}_{2} \mathrm{O}_{3}$-Ce:GdYAG composite ceramic phosphors for high-power white light-emitting-diode applications. Journal of the European Ceramic Society, 2019, 39(6): 2149-2154.

[53] CHEN J, TANG Y, YI X, et al. $\mathrm{Al}_{2} \mathrm{O}_{3}-\mathrm{Ce}_{2} \mathrm{~Tb}_{3} \mathrm{Al}_{5} \mathrm{O}_{12}$ composite ceramic phosphors for high efficiency warm white light illumination. Optical Materials, 2019, 97: 109384.

[54] TIAN Y, TANG Y, YI X, et al. Study of composite $\mathrm{Al}_{2} \mathrm{O}_{3}-\mathrm{Ce}$ : $\mathrm{Y}_{3} \mathrm{Mg}_{1.8} \mathrm{Al}_{1.4} \mathrm{Si}_{1.8} \mathrm{O}_{12}$ ceramic phosphors. Opt. Lett., 2019, 44(19): 4845-4848.

[55] ZHENG R, LUO D, YUAN Y, et al. $\mathrm{Dy}^{3+} / \mathrm{Ce}^{3+}$ codoped YAG transparent ceramics for single-composition tunable white-light phosphor. Journal of the American Ceramic Society, 2015, 98(10): 3231-3235.

[56] HU S, LU C H, QIN X P, et al. Color tuning of $\mathrm{Lu}_{3} \mathrm{Al}_{5} \mathrm{O}_{12}: \mathrm{Dy}^{3+}$ ceramic-based white light-emitting phosphors via $\mathrm{Yb}$ incorporation. Journal of the European Ceramic Society, 2017, 37(1): 229-237.

[57] WANG L, XIE R J, SUEHIRO T, et al. Down-conversion nitride materials for solid state lighting: recent advances and perspectives. Chemical Reviews, 2018, 118(4): 1951-2009.

[58] LI S X, ZHU Q Q, WANG L, et al. $\mathrm{CaAlSiN}_{3}: \mathrm{Eu}^{2+}$ translucent ceramic: a promising robust and efficient red color converter for solid state laser displays and lighting. Journal of Materials Chemistry C, 2016, 4(35): 8197-8205.

[59] JOSHI B, HOON J S, KSHETRI Y K, et al. Transparent Sialon phosphor ceramic plates for white light emitting diodes applications. Ceramics International, 2018, 44(18): 23116-23124.

[60] JOSHI B, KSHETRI Y K, GYAWALI G, et al. Transparent $\mathrm{Mg}-\alpha / \beta$-Sialon: $\mathrm{Eu}^{2+}$ ceramics as a yellow phosphor for pc-WLED. Journal of Alloys and Compounds, 2015, 631: 38-45.

[61] LI K, WANG H, LIU X, et al. $\mathrm{Mn}^{2+}$ activated MgAlON transparent ceramic: a new green-emitting transparent ceramic phosphor for high-power white LED. Journal of the European Ceramic Society, 2017, 37(13): 4229-4233.

[62] PRICHA I, ROSSNER W, MOOS R. Pressureless sintering of luminescent $\mathrm{CaAlSiN}_{3}:$ Eu ceramics. Journal of Ceramic Science and Technology, 2015, 6(1): 63-67.

[63] RAUKAS M, KELSO J, ZHENG Y, et al. Ceramic phosphors for light conversion in LEDs. ECS Journal of Solid State Science and Technology, 2013, 2(2): R3168-R3176.

[64] WIEG A T, PENILLA E H, HARDIN C L, et al. Broadband white light emission from Ce:AlN ceramics: high thermal conductivity down-converters for LED and laser-driven solid state lighting. Appl. Materials, 2016, 4(12): 126105.

[65] SCHNICK W. Shine a light with nitrides. Physica Status Solidi (RRL)-Rapid Research Letters, 2009, 3(7/8): A113-A114.

[66] XIE R J, HIROSAKI N, SUEHIRO T, et al. A simple, efficient synthetic route to $\mathrm{Sr}_{2} \mathrm{Si}_{5} \mathrm{~N}_{8}$ : $\mathrm{Eu}^{2+}$-based red phosphors for white lightemitting diodes. Chemistry of Materials, 2006, 18(23): 5578-5583.

[67] LI S X, WANG L, TANG D M, et al. Achieving high quantum efficiency narrow-band $\beta$-Sialon: $\mathrm{Eu}^{2+}$ phosphors for high-brightness $\mathrm{LCD}$ backlights by reducing the $\mathrm{Eu}^{3+}$ luminescence killer. Chemistry of Materials, 2018, 30(2): 494-505.

[68] XIE R J, HIROSAKI N, MITOMO M, et al. Wavelength-tunable and thermally stable $\mathrm{Li}-\alpha$-sialon: $\mathrm{Eu}^{2+}$ oxynitride phosphors for white light-emitting diodes. Applied Physics Letters, 2006, 89(24): 241103.

[69] ZHANG Y, LIU Y, YANG L, et al. Preparation and luminescence properties of thermally stable $\mathrm{Mn}^{4+}$ doped spinel red-emitting ceramic phosphors. Journal of Luminescence, 2020, 220: 117016.

[70] ARREDONDO A, DESIRENA H, MORENO I, et al. Dual color tuning in $\mathrm{Ce}^{3+}$-doped oxyfluoride ceramic phosphor plate for white LED application. Journal of the American Ceramic Society, 2019, 102(3): $1425-1434$.

[71] HU S, LIU Y, ZHANG Y, et al. 3D printed ceramic phosphor and the photoluminescence property under blue laser excitation. Journal of the European Ceramic Society, 2019, 39(8): 2731-2738. 